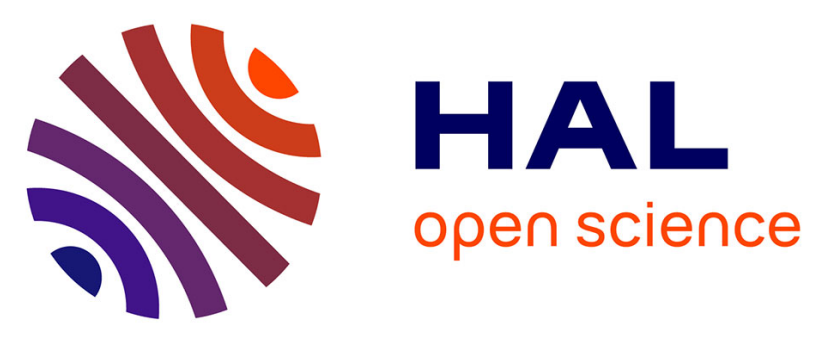

\title{
Enquête sur la prise en charge des chocs anaphylactiques aux curares auprès des anesthésistes réanimateurs de la région Picardie : de la déclaration à la consultation d'allergologie
}

\author{
Sophie Romby
}

\section{To cite this version:}

Sophie Romby. Enquête sur la prise en charge des chocs anaphylactiques aux curares auprès des anesthésistes réanimateurs de la région Picardie: de la déclaration à la consultation d'allergologie. Médecine humaine et pathologie. 2015. dumas-01229021

\section{HAL Id: dumas-01229021 https://dumas.ccsd.cnrs.fr/dumas-01229021}

Submitted on 4 Dec 2015

HAL is a multi-disciplinary open access archive for the deposit and dissemination of scientific research documents, whether they are published or not. The documents may come from teaching and research institutions in France or abroad, or from public or private research centers.
L'archive ouverte pluridisciplinaire HAL, est destinée au dépôt et à la diffusion de documents scientifiques de niveau recherche, publiés ou non, émanant des établissements d'enseignement et de recherche français ou étrangers, des laboratoires publics ou privés. 


\section{UNIVERSITE DE PICARDIE JULES VERNE}

FACULTE DE MEDECINE

ANNEE 2015

$\mathrm{N}^{\circ} 2015-37$

Enquête sur la prise en charge des chocs anaphylactiques aux curares auprès des anesthésistes réanimateurs de la région Picardie: de la déclaration à la consultation d'allergologie

THESE POUR LE DOCTORAT EN MEDECINE

Présentée et soutenue publiquement

Le 23 avril 2015

Par SOPHIE ROMBY

Président du Jury :

Monsieur le Professeur Hervé DUPONT

Juges:

Monsieur le Professeur Pierre ANDREJAK

Monsieur le Professeur Pascal BERNA

Monsieur le Docteur Yazine MAHJOUB

Directeur de thèse : 


\section{TABLE DES MATIERES}

Introduction $\quad 11$

$\begin{array}{ll}\text { Matériel et méthode } & 13\end{array}$

$\begin{array}{ll}\text { Résultats } & 14\end{array}$

$\begin{array}{ll}\text { Discussion } & 24\end{array}$

$\begin{array}{ll}\text { Conclusion } & 34\end{array}$

$\begin{array}{ll}\text { Bibliographie } & 35\end{array}$

$\begin{array}{ll}\text { Annexes } & 39\end{array}$ 
A mon Maître et président de thèse,

\section{Monsieur le Professeur Hervé DUPONT}

Professeur des Universités-Praticien Hospitalier

Anesthésie-Réanimation

Chef du service "Anesthésie-Réanimation"

Co-chef du Pôle "Anesthésie-Réanimation, médecine d'urgence"

Vous m'avez accueillie dans votre spécialité,

J'ai bénéficié d'une formation de qualité, reconnue partout où je vais

Vous me faites l'honneur de juger ce travail,

Que celui-ci soit l'occasion de vous témoigner ma gratitude et mon profond respect. 


\section{Monsieur le Professeur Michel ANDREJAK}

Professeur des Universités-Praticien Hospitalier

Pharmacologie fondamentale clinique

Directeur du Centre Régional de Pharmacovigilance d'AMIENS

Responsable du service de pharmacologie clinique

Pôle Biologie, Pharmacie et Santé des populations

Officier dans l'Ordre des Palmes Académiques

Vous m'avez ouvert les portes de la pharmacovigilance.

Vous me faîtes l'honneur de juger ce travail.

Que cette thèse soit le témoignage de ma profonde reconnaissance et de mon plus grand respect. 


\section{Monsieur le Professeur Pascal BERNA}

Professeur des Universités - Praticien Hospitalier

Responsable du service de chirurgie thoracique

Pôle « Cœur - Thorax - Vaisseaux »

Travailler à tes cotés m'a beaucoup appris.

Tu as su monter un service avec une équipe soudée où règne une ambiance familiale. Mais il ne faut pas s'y tromper, travail, rigueur et innovation en font partie intégrante. C'est pour ce genre de services que le secteur hospitalier prend du sens. Merci d'avoir accepté au pied levé de faire partie de mon jury de thèse. 


\section{Monsieur le Docteur Yazine MAHJOUB}

Maître de Conférences des Universités - Praticien Hospitalier

Anesthésie-Réanimation

Ta culture médicale m'impressionne,

Ta gestion des urgences vitales encore plus : calme, sang-froid et rigueur.

Pour autant tu sais rester accessible pour ton équipe.

Tu mérites de gravir vite les échelons, sans jamais avoir le vertige.

J'espère pouvoir continuer à te demander conseil en cas de besoin et mettre un coup de râteau dans le jardin japonais. 


\section{Madame le Docteur Stéphanie MALAQUIN}

Chef de clinique des universités-assistant des hôpitaux

Anesthésie-Réanimation

Première rencontre lors d'un apéro d'orthopédistes,

Puis comme co-interne en chirurgie viscérale,

Puis comme chef et pour finir comme directrice de thèse,

Rigueur et perfectionnisme,

Qualités acquises en danse classique et mises en application en réanimation

Cela peut parfois impressionner, mais il ne faut pas se méprendre,

Disponibilité, écoute et fiabilité sont aussi au rendez-vous,

Merci d'avoir accepté de diriger mon travail, même si je ne suis pas la thésarde parfaite. 


\section{A ma famille}

A mes parents,

Vous m'avez accompagné même de loin tout le long de mes études ainsi qu'à chaque petit accident de la vie.

A ma mère, il paraît que tout se joue avant l'âge de 6 ans...ou peut-être même 4 . Merci pour ton éducation. A mon père, pour m'avoir appris à être autonome dès le plus jeune âge mais aussi pour être toujours dispo pour donner un conseil de gestion en tout genre (comptes en banque, impôts, gestion du personnel....ça tombe bien je n'ai jamais eu de cours dans ces domaines).

\section{A mes frères,}

Pas facile de cohabiter avec une petite princesse. Merci à François pour ta patience et ta gentillesse. A Pierre, il y a 12 ans je te traumatisais en bossant ma P1. Finalement te voilà parti dans les joies de cette année mythique. Je crois en toi !

A mes grands-parents,

Mamé et Papé, malheureusement vous ne pouvez pas être présents. Mamé, même si tu ne sais plus ce qu'est une thèse, je suis sûre que tu aurais été fière de moi.

A ma belle-famille,

A Martine, pour m'avoir accueilli lors d'un moment difficile.

A Aurélie, chirurgien de talent, j'ai hâte de travailler en famille.

A Julien ......Merci de me faire mes certificats d'aptitude même à contrecœur.

A Simon......le meilleur pour la fin ;)

Merci de supporter chaque petite lubie sans broncher et de me canaliser. Tu m'as apporté stabilité tout en me laissant libre de voler à mes différents projets. Je t'aime. 


\section{A mes amis,}

$$
\text { D'école }
$$

Sophie, Aurélie, la distance est là mais je ne vous oublie pas

$$
\text { De la fac de médecine de Marseille, }
$$

Florent et Alexandre tout d'abord. Premiers amis rencontrés sur un banc de l'amphi Propé.

A Mawie toutcour, rencontre à la cafet pendant un cours de master d'anat appliquée. Que de fous rires pendant les soirées !

A Perrine, le globe-trotteur qui voyage aux 4 coins du monde, même à Ômiens.

A Aurélia, alias Stone ma marraine de cœur. Le clic-clac toujours dispo. Toujours prête pour un bon petit repas maison ou pour dévorer un plateau de sushi.

\section{Du DESAR d'AMIENS,}

A mes co-internes (qui m’ont lâchement abandonné)

Clala, le coach sportif. La course du maroilles, ça va être chaud mais ce n'est que partie remise.

A Amel et Jess, qui m’ont permis de survivre à la réa chir,

A Vincent, Paola, Marion, Sandra, Léonie, Youssef, Quentin, sans qui cet internat n'aurait pas été le même.

A Medhi et Vincent, les compagnons de galère, ça y est c'est fini !!

A Abdel, sponsor officiel de mon semestre de dispo. Combien de gardes tu m'as repris ??

A Kahina et Stéphane, pas mieux.

A tous les autres DESAR

A Nathalie, Mélody, pour pouvoir parler d'autre chose que de chirurgie.

Aux chirurgiens orthopédiques, du membre inf, membre sup, du petit orteil gauche, il faut revoir vos délais de consolidation.

A Hélène, Juliette, Morgane, Florine, Lucie, Mathilde \& Mathilde, Chloé, Léa, Elise, Vincent, Mathurin, Gautier, Benoît ,Florent, David, Maxime, Gaëtan, Xavier, merci de m'avoir accueillis à Amiens.

Aux skypotos et skygreluches, à bientôt dans les airs. 


\section{A mes collègues,}

$$
\text { De St-Q }
$$

A Yves Pila qui m'a appris mes premiers gestes.

A Bernard Cassett', qui m'a montré qu'on pouvait être un vrai chef de service et anesthésiste, et qu'il était possible d'endormir plus de 4 patients par jour. Ton expérience ou mon âge.... J'espère un jour avoir la même dextérité !

A Jean-Mi, preuve vivante qu'on peut être toujours passionné par son métier et innover même après une longue carrière (mais loin d'être terminée).

A Etienne, qui tel un chat retombe toujours sur ses pattes.

Et bien sûrs à tous les IADE et IDE, pour m'avoir appris tout le travail de l'ombre de l'anesthésie.

A Delphine Olory, pour le soutien lors de mes premières nuits à la mater. Tu m'as fait changer de vision sur les sages-femmes.

\section{D’Abbeville}

$\mathrm{Au}$ docteur Clabaut, malheureusement parti trop vite, pour son regard bienveillant.

Au Dr Souissi et au Dr Méchaouri, pour votre patience.

\section{De Beauvais}

A Mme Guerin, calme, compétente, rigueur et charisme. J'espère un jour vous ressembler.

Au docteur Marienne, vous avez su donner une impulsion à la réanimation, où les internes se pressaient pour y passer en stage.

\section{$\mathrm{Du} C H U$,}

A mes nombreux chefs de terrain de stage, croisés en garde : au docteur Z, à Stéphanie Suzanne, Edouard, Evgeni et Raluca, Rachid, Ivan et Volker, Vincent, Philippe, Ghada et Edouard, au Docteur Montpellier et Andréi, Radhi, Pierre, Adel, Beatris, un grand merci.

A Christophe, comme quoi il ne faut pas écouter les commérages, merci de m'avoir transmis un peu de ton expérience. 
A Mélanie Perquin et au Docteur Benabes, merci d'avoir répondu à mes questions.

A tous les IADE du bloc même ceux de l'ancien sud qui ont su m'accompagner et me faire confiance.

Aux IADE, IDE, CCA du SAMU, pour m'avoir fait découvrir la campagne picarde. Les pilotes je ne vous remercie pas, je n'ai toujours pas été à la mer.

D'ailleurs

Aux nombreux MAR de clinique du 80 et d'ailleurs, qui sans être passés par la filière universitaire sont aussi capables d'accompagner et affiner la formation : Romain, Alice, Kathy, Philippe, Stéphanie, Marek, François, au docteur Clais : merci. 


\section{INTRODUCTION}

Plus de 200 millions de chirurgies sont réalisées dans le monde chaque année (1). Des études récentes montrent que la morbi-mortalité péri-opératoire reste significative (2). L'anesthésie ne fournit pas de bénéfice direct au patient et chaque médecin anesthésisteréanimateur (MAR) tend à analyser les risques pour mieux les maîtriser. Cette analyse débouche sur le choix d'une technique anesthésique adaptée à chaque patient et à l'acte opératoire prévu.

Le choc anaphylactique (CA) est une complication anesthésique rare mais qui reste redoutée. Il représente de 9 à 19\% des complications liées à l'anesthésie suivant les pays (3). Les réactions d'hypersensibilité immédiate en anesthésie générale et locorégionale ont été évaluées à 1/13000 en 1996. L’incidence des réactions allergiques est de 1/5500 anesthésies et augmente même jusqu'à 1/4000 chez la femme en France (4,5). Elle est de 1/3500 anesthésies en Grande Bretagne (6), de 1/6000 en Norvège (7).

De nombreux produits utilisés en anesthésie peuvent être responsables d'une réaction allergique. Le produit le plus souvent incriminé est représenté par la famille des curares (55\% des cas) (8), puis le latex $(22.3 \%)$ et les antibiotiques $(14.7 \%)(9,10)$.

La mortalité due à une réaction allergique aux curares varie suivant le pays de 3 à $9 \%$ (11-13). Elle reste importante même lorsque le traitement est entrepris rapidement et ne dépend pas du type de curare utilisé (14). La morbidité est principalement représentée par les encéphalopathies anoxiques, dont l'incidence n'est pas chiffrée (15).

Les recommandations de la SFAR-SFA de 2002 sur la «prévention du risque d'allergique per-anesthésique » préconisent une investigation immédiate et à distance pour confirmer le diagnostic et ainsi adapter une anesthésie ultérieure (16). L'anesthésisteréanimateur doit s'assurer de la mise en œuvre de d'un prélèvement biologique comportant un dosage d'histamine et de tryptase sériques, d'IgE spécifiques et d'un dosage de leucotriènes urinaires (17).

Dans un deuxième temps, une consultation d'allergologie doit être réalisée. Elle permet de confirmer le diagnostic et de rechercher des allergies croisées (18-20). Son objectif est de diminuer la morbi-mortalité (11), en permettant de choisir le curare adapté lors une anesthésie ultérieure (21). 
Une réaction allergique à un médicament constitue un effet indésirable qu'il convient de déclarer immédiatement au centre régional de pharmacovigilance (CRP).

Le système national de pharmacovigilance mis en place dès les années 1970 est un réseau de 31 CRP (22), dont les rôles sont d'évaluer les effets indésirables des médicaments une fois l'AMM obtenue $(23,24)$ d'en déterminer la prévalence et les facteurs de risques grâce à des enquêtes nationales $(25,26)$.

La notification au CRP par le MAR qui constate la réaction allergique per-anesthésique est donc capitale.

Malgré les recommandations, seulement $65 \%$ des patients suspectés bénéficient d'une consultation allergologie et donc d'un diagnostic final (27). De plus, bien que rendue obligatoire par le législateur par le décret de 1984 (28), il existe une sous déclaration des CA même si aucune étude n'est disponible sur le sujet. Il y a donc un risque important de méconnaitre une allergie aux curares et de réexposer le patient par erreur lors d'une anesthésie ultérieure.

L'objectif de cette étude est d'évaluer, à l'échelon régional, la qualité de la déclaration par les anesthésistes des CA supposés aux curares et l'accompagnement du patient jusqu'au diagnostic positif d'allergie. 


\section{MATERIEL ET METHODE}

Il s'agit d'une étude prospective descriptive observationnelle par analyse d'un questionnaire anonyme envoyé à tous les MAR travaillant en centre hospitalier de la région Picardie portant sur la déclaration du CA peropératoire supposé aux curares.

\section{$\underline{\text { REALISATION DU QUESTIONNAIRE }}$}

Le questionnaire est constitué de 7 items et de 23 questions.

Le $1^{\text {er }}$ item concernait des données épidémiologiques : ancienneté et lieu d'exercice (CHU/CHG). Les réponses à ces questions étaient ciblées et les réponses multiples n'étaient pas possibles.

Le $2^{\text {ème }}$ item concernait la fréquence à laquelle le praticien a été confronté à un choc anaphylactique. La première question attendait une réponse ciblée (OUI : a déjà été confronté à un CA / NON : n'a jamais été confronté). Les MAR qui n'y ont jamais été confrontés ne pouvaient pas répondre à la suite du questionnaire. La deuxième question concernait le nombre de CA en fonction du grade (grade 1 à 4 de la classification de Ring et Messner) (29).

Le $3^{\text {ème }}$ item concernait la déclaration en pharmacovigilance et était composée de 8 questions portant sur la fréquence des déclarations en fonction du grade, des horaires des prélèvements allergiques, de la procédure utilisée pour la déclaration ainsi que les pièces fournies.

Le $4^{\text {ème }}$ item concernait l'information orale et écrite délivrée au patient.

Le $5^{\text {ème }}$ item concernait l'obtention des résultats biologiques et du compte rendu de la consultation d'allergologie, ainsi que son délai de réalisation.

Le $6^{\text {ème }}$ item comportait 6 questions sur la filière d'allergo-anesthésie.

Le $7^{\text {ème }}$ item concernait les modalités de la consultation pré-anesthésique (CPA) d'un patient allergique. Elle comportait 2 questions sur la procédure à suivre pour obtenir la confirmation de l'allergie. 


\section{DIFFUSION DU QUESTIONNAIRE}

Le questionnaire a été envoyé aux MAR de Picardie par courriel, sous forme d'un document Google ${ }^{\circledR}$ doc à renvoyer. La mailing-list des MAR exerçant en CHU a été obtenue auprès du secrétariat du coordonnateur de Picardie au CHU d'Amiens. Celle des MAR exerçant en CHG a été réactualisée en contactant chaque secrétariat d'anesthésie. Il n'a pas été tenu compte des MAR intérimaires. Les MAR des CHG étaient répartis sur les 3 départements regroupant les CHG d'Abbeville, Beauvais, Creil, Compiègne, Doullens, Château-Thierry, Chauny, Laon, Péronne, Senlis, Soissons et St-Quentin.

Le questionnaire a été envoyé la première fois le 11 février 2015, avec deux rappels à quinze jours d'intervalle.

\section{$\underline{\text { RECUEIL ET ANALYSE DES DONNEES }}$}

Pour chaque MAR répondant, les données étaient traitées en temps réel par le logiciel d'exploitation de Google $®$ doc. Les résultats apparaissaient sous un format « liste », créant pour chaque question un tableau à double entrée dont l'abscisse représentait les réponses possibles et l'ordonnée les intitulés de chaque question.

Les répondeurs ont été divisés en 2 groupes :

- MAR CHU: représentant les MAR travaillant au CHU d'Amiens.

- MAR CHG : représentant les MAR travaillant en CHG dans la région Picardie.

Les résultats sont exprimés en valeurs absolues et pourcentages de répondants. Les comparaisons entre les groupes concernant les variables qualitatives ont été effectuées par le test non paramétrique de Chi 2out de Fisher en cas de petits effectifs. Les répartitions des variables ordinales ont été comparées grâce au test non paramétrique de Kruskall Wallis. La différence était considérée comme significative pour une valeur de $\mathrm{p}$ inférieure à 0,05 . L'analyse statistique a été menée par la plateforme en ligne du logiciel R ®. 


\section{RESULTATS}

122 MAR ont été contactés parmi lesquels 50 MAR de CHU et 72 de CHG. 78 ont répondu (33 au CHU et 45 en $\mathrm{CHG}$ ), permettant un taux de participation de 64\% entre le 11 février 2015 et le 20 mars 2015.

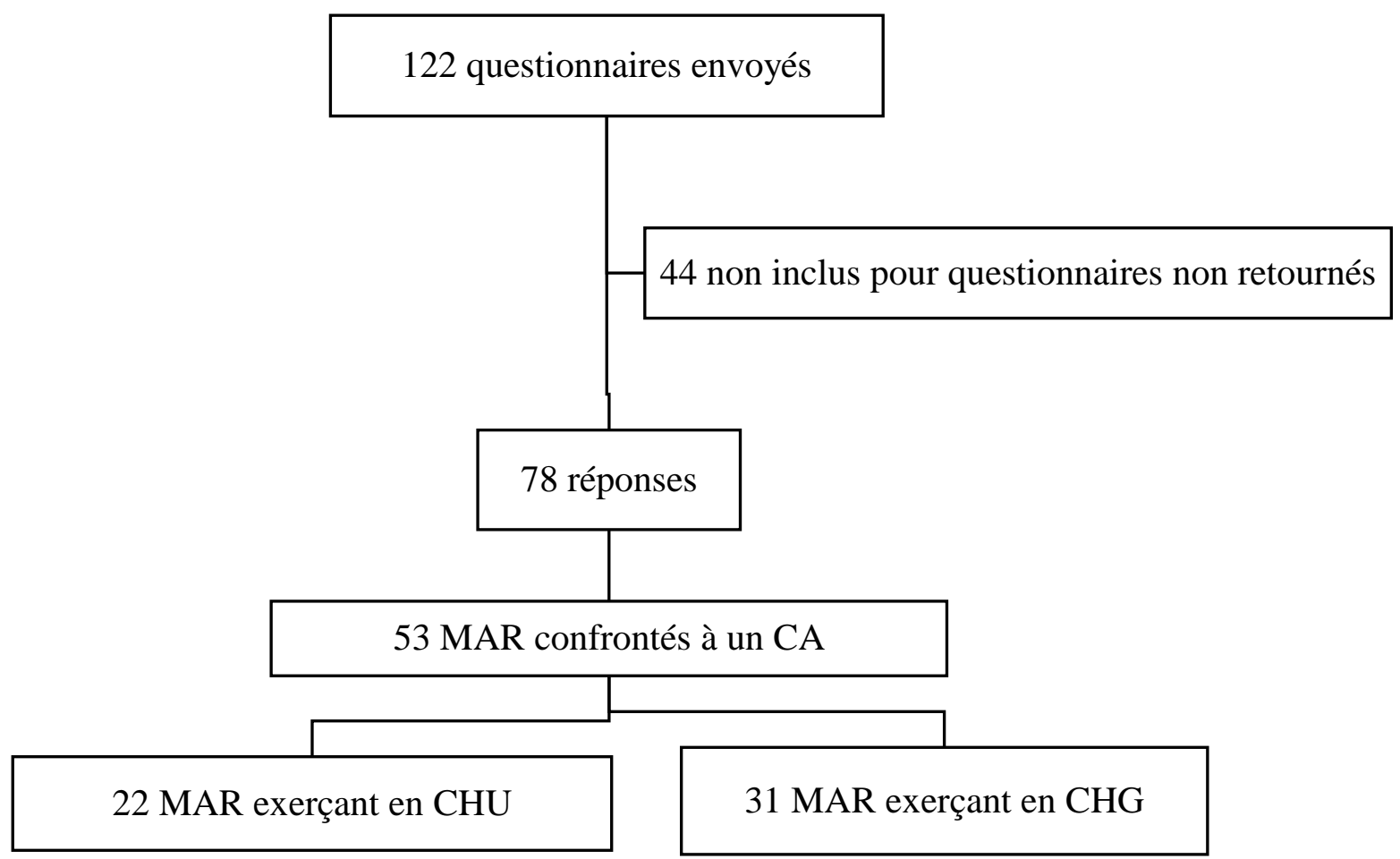

Figure 1 : Diagramme en flux relatif aux taux de réponses en fonction du lieu d'exercice. CA :

Choc anaphylactique. MAR = Médecin anesthésiste réanimateur.

Les données démographiques des répondants sont représentées dans le tableau 1.

Tableau 1 : Données démographiques des répondants selon leur lieu d'exercice.

\begin{tabular}{cccc}
\hline & $\begin{array}{c}\text { CHU } \\
\mathbf{n = 3 3}\end{array}$ & $\begin{array}{c}\text { CHG } \\
\mathbf{n = 4 5}\end{array}$ & $\mathrm{p}$ \\
\hline Années d'exercice & & & \\
$<2$ ans & $8(24)$ & $4(9)$ & 0.11 \\
$\leq 10$ ans & $12(36)$ & $6(13)$ & 0.03 \\
$>10$ ans & $13(39)$ & $35(78)$ & 0.001 \\
\hline
\end{tabular}

Les variables sont exprimées en valeurs absolues (\%) 
Les modalités d'exercice des MAR ayant été confrontés à un choc sont détaillées dans le tableau 2.

Tableau 2 : Données démographiques des répondants et modalités d'exercice.

\begin{tabular}{cccc}
\hline & $\begin{array}{c}\text { Confronté à un } \\
\text { choc } \\
\mathbf{n = 5 3}\end{array}$ & $\begin{array}{c}\text { Non confronté } \\
\text { à un choc } \\
\mathbf{n = 2 5}\end{array}$ & $\mathrm{p}$ \\
\hline Lieu d'exercice & $22(42)$ & $11(44)$ & 0.14 \\
CHU & $31(58)$ & $14(56)$ & 0.14 \\
CHG & & & \\
& & & \\
Années d'exercice & $7(13)$ & $5(20)$ & 0.51 \\
$<2$ ans & $10(19)$ & $8(32)$ & 0.25 \\
$\leq 10$ ans & $36(68)$ & $12(50)$ & 0.13 \\
\hline 10 ans & & &
\end{tabular}

Les variables sont exprimées en valeurs absolues (\%).

Tous stades confondus, $32(41 \%)$ MAR ont déjà été confrontés à plus de 5CA. La répartition et la sévérité des CA rapportés par les MAR depuis le début de leur carrière sont détaillées dans la figure 2 . 


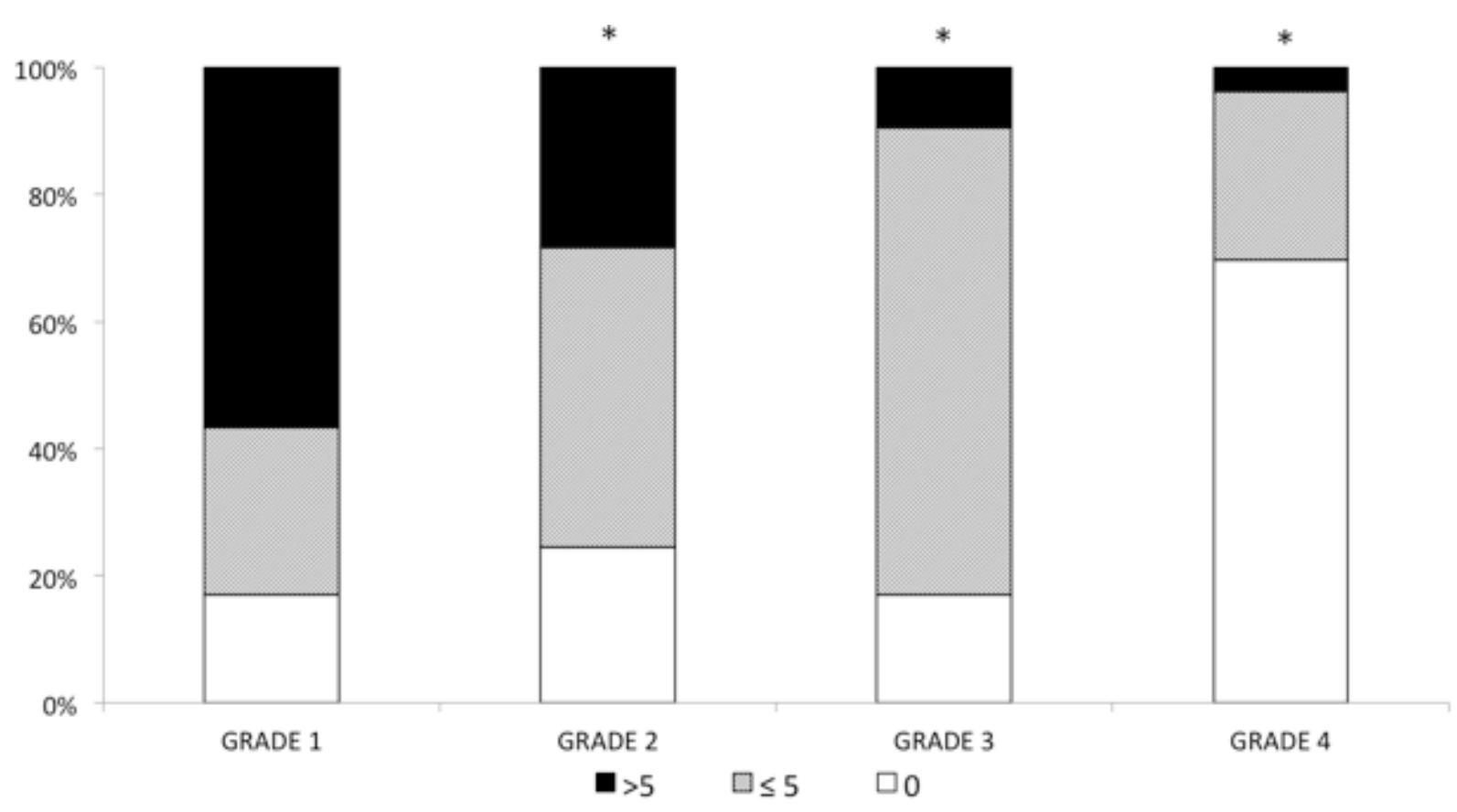

Figure 2 : Répartition du nombre de chocs anaphylactiques rapportés par les MAR interrogés en fonction des grades de Ring et Messmer(29). * $=\mathrm{p} \leq 0.005$ vs grade 1 .

Trente-huit (71\%) MAR ayant déjà traités des grades 3 ou 4 ont également pris en charge des CA de grade inférieur.

\section{DECLARATION DE PHARMACOVIGILANCE}

Neuf (17\%) MAR déclarent « toujours » leurs chocs tous stades confondus dont 4 (18\%) MAR de CHU et 5 (16\%) MAR de CHG. La fréquence de la déclaration en fonction du grade est détaillée dans la figure 3. 


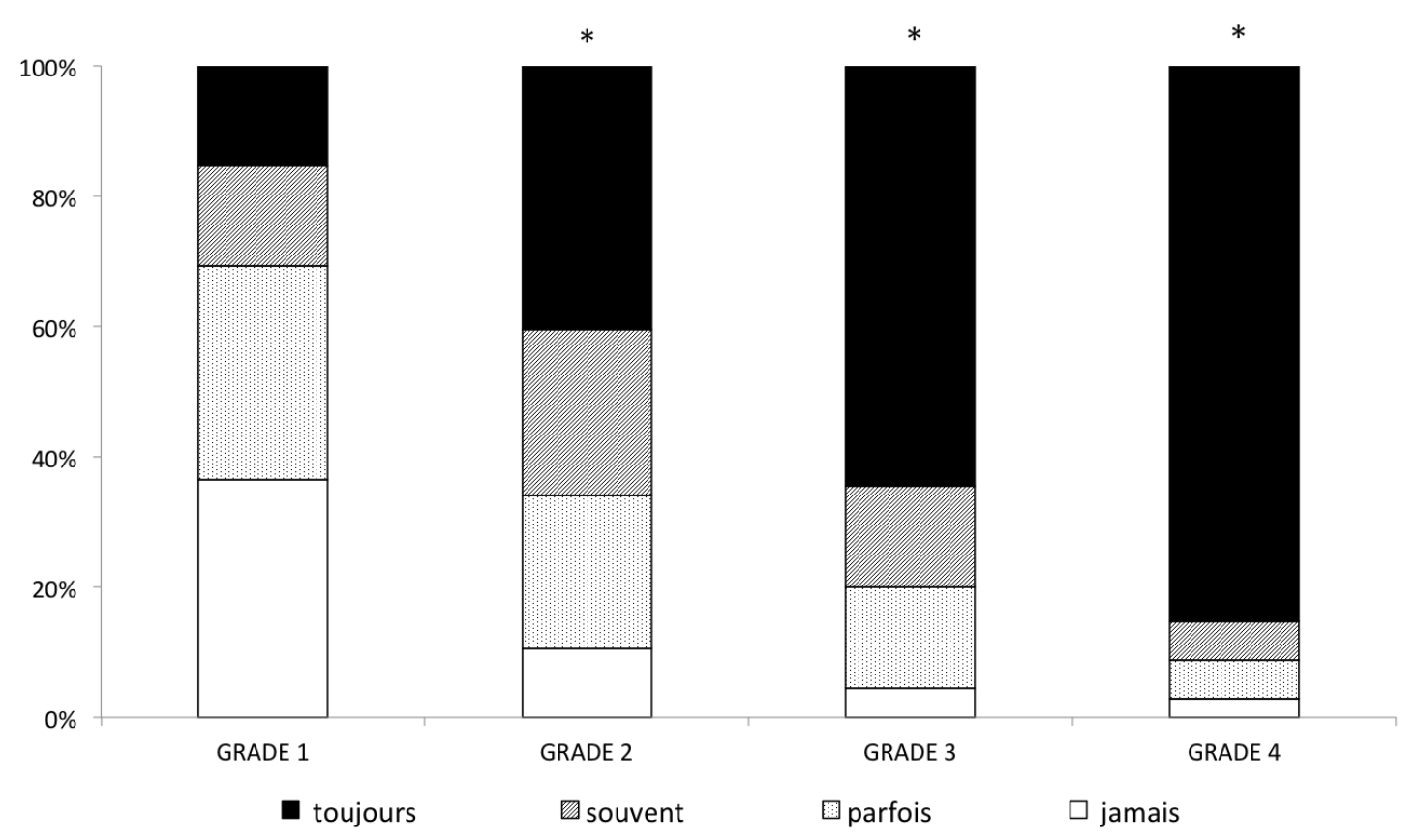

Figure 3 : Fréquence de déclaration de pharmacovigilance en fonction des grades de Ring et Messmer (29). ${ }^{*}=\mathrm{p} \leq 0.005$ vs grade 1 .

\section{$\underline{\text { MODALITES DE LA DECLARATION }}$}

Les modalités de déclaration suivant le site d'exercice sont décrites dans les figures 4 et 5.

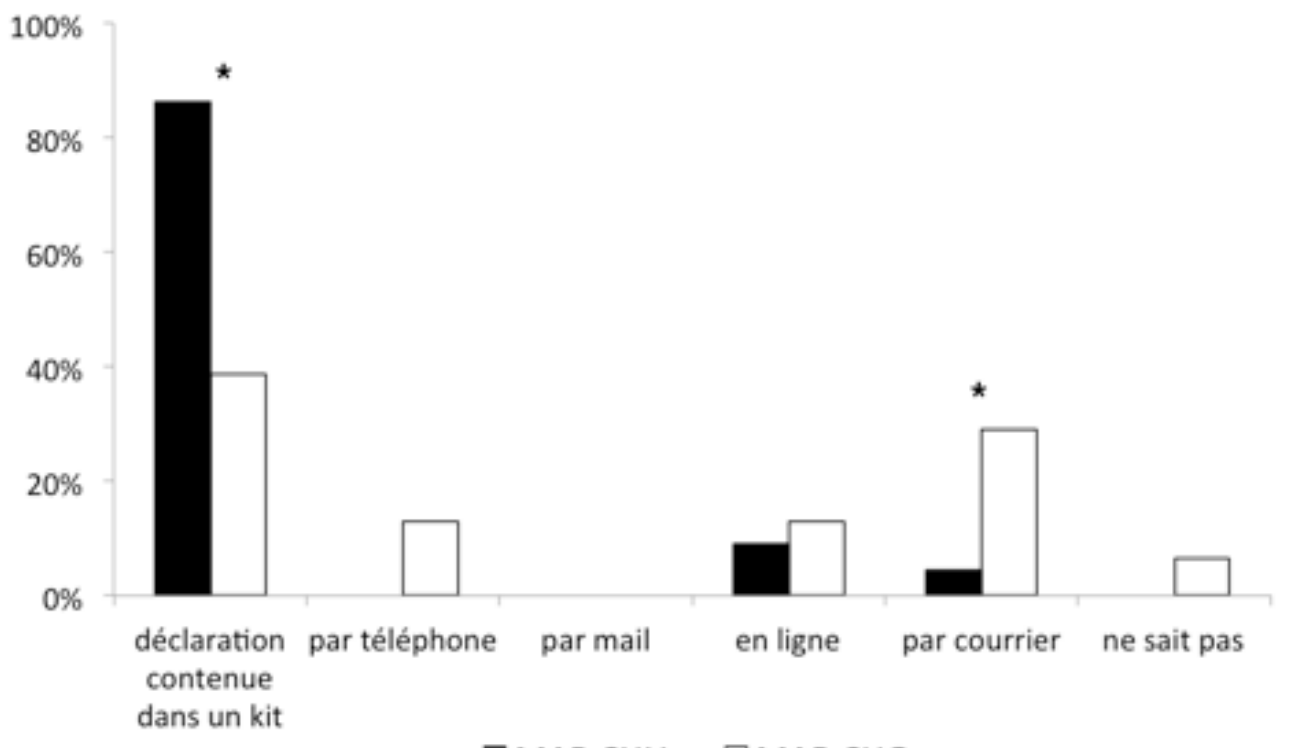

- MAR CHU $\square$ MARCHG

Figure 4 : Modalités de la déclaration suivant le site d'exercice. ${ }^{*}=p \leq 0.005$ 


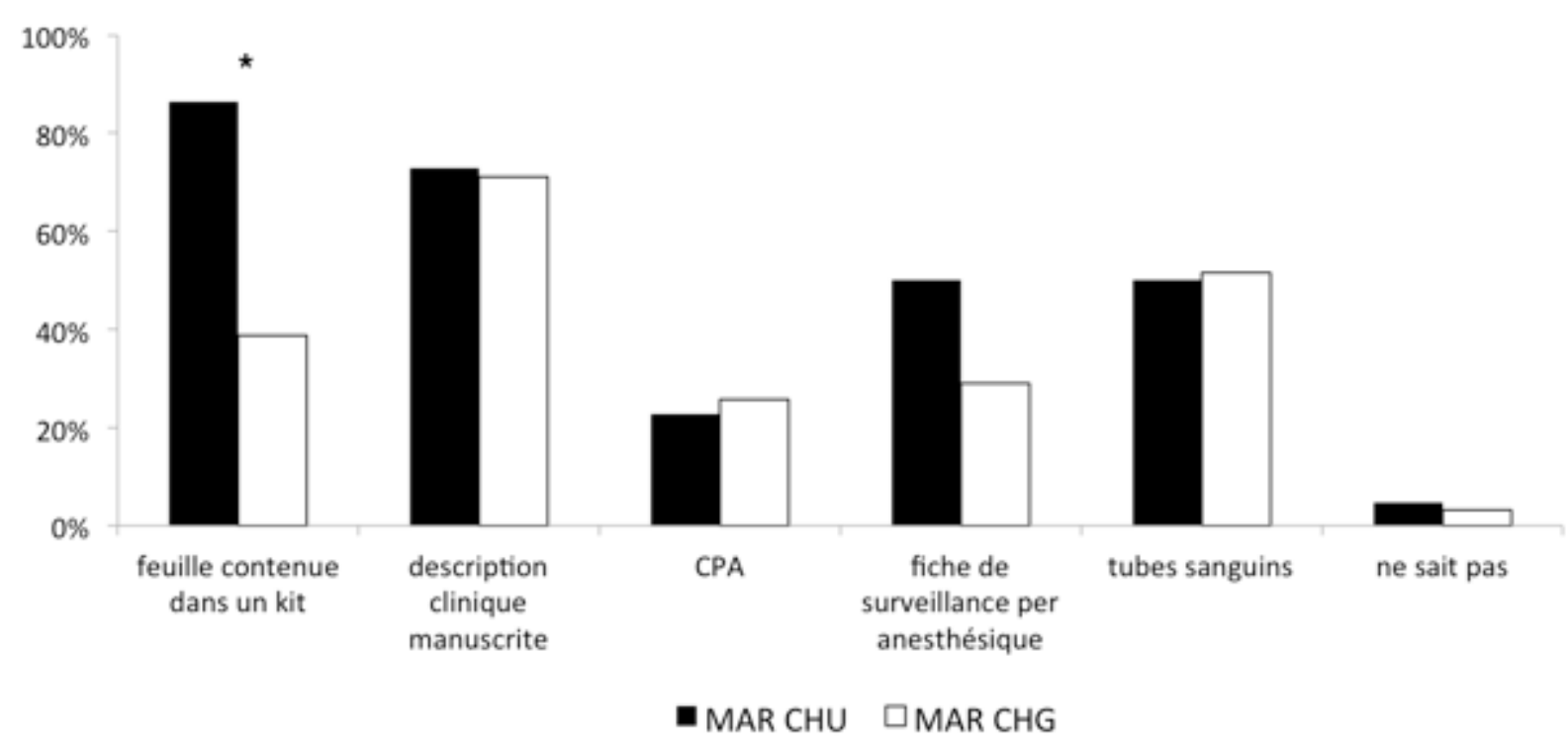

Figure 5 : Pièces fournies lors de la déclaration

Trois (10\%) MAR de CHG ne fournissent aucune lettre décrivant l'évènement (ni feuille présente dans le kit ni description manuscrite).

\section{INVESTIGATIONS PER ET POST-OPERATOIRES IMMEDIATES}

Quarante-six (87\%) MAR effectuent un prélèvement de tryptase et d'histamine la 1 ère heure. Les MAR répètent le prélèvement de tryptase et d'histamine à $\mathrm{H} 2$ respectivement dans $53 \%$ et $51 \%$ des cas .Dix-sept (32\%) MAR répètent le dosage d'histamine plus de $3 \mathrm{~h}$ après le début du CA.

Trente et un (58\%) MAR font le dosage d'IgE spécifiques la première heure. Onze (21\%) MAR les dosent la $2^{\text {ème }}$ heure, 6 (11\%) les dosent à H3, 14 (27\%) les dosent entre H12-H24.

Neuf (17\%) MAR font deux prélèvements d’IgE spécifiques.

Trois (6\%) MAR dosent les leucotriènes urinaires la $1^{\text {ère }}$ heure, aucun la seconde, 16 $(31 \%)$ la $3^{\text {ième }}$ heure et $22(42 \%)$ entre 12 à $24 \mathrm{~h}$ après le CA. Dix-huit $(35 \%)$ ignorent l'horaire de prélèvement. Cinq (9\%) MAR font 2 prélèvements, un à H3 et l'autre entre 12 et $24 \mathrm{~h}$. 


\section{INFORMATION DU PATIENT}

Le contenu de l'information orale donnée au patient est détaillé dans la figure 6.

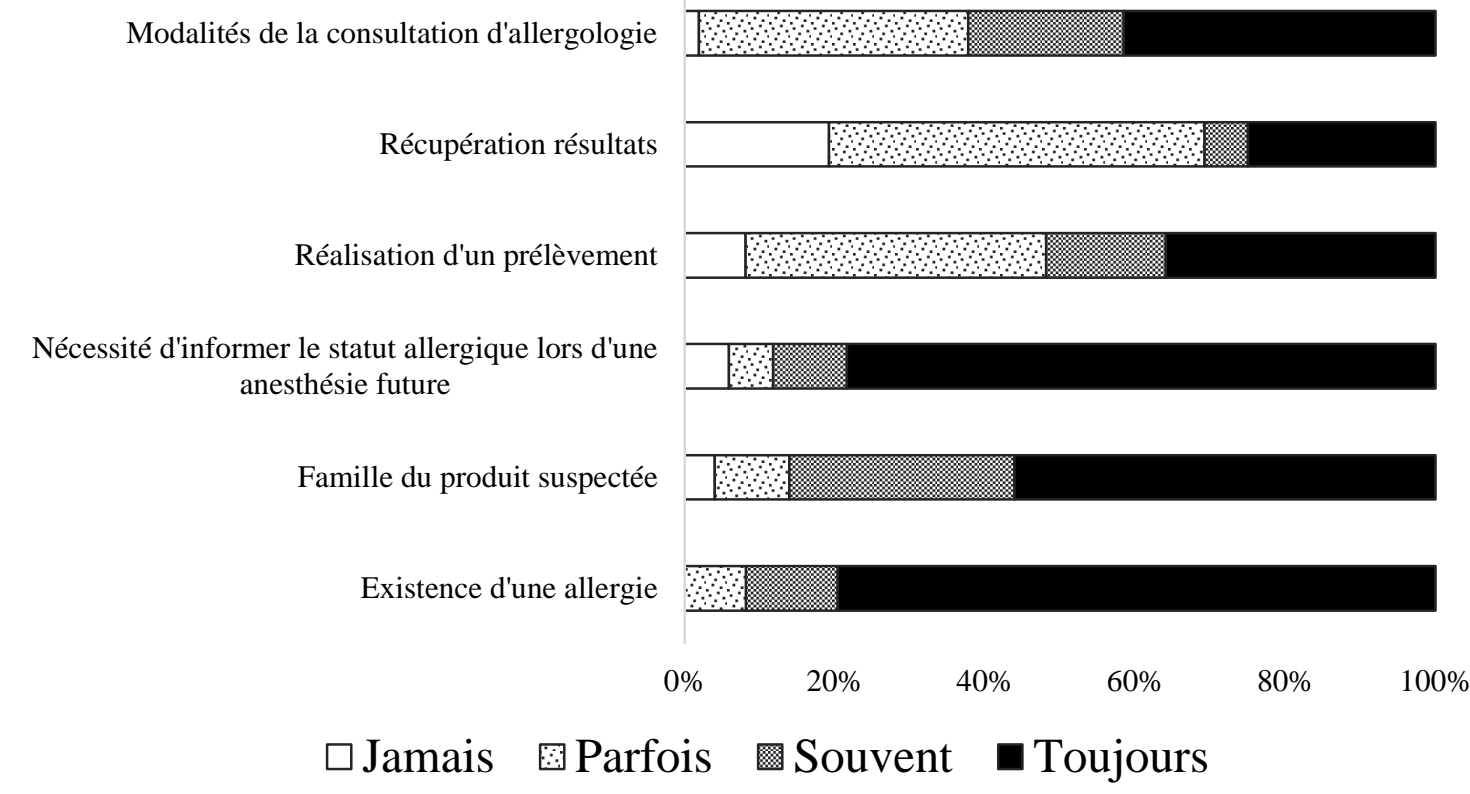

Figure 6 : contenu de l'information donnée au patient.

Cinquante et un (96\%) MAR donnent personnellement l'information de l'allergie.

Trente-quatre (64\%) informent le patient en SSPI et 39 (79\%) en service. Dix-neuf (36\%) MAR donnent l'information deux fois, en SSPI et en service. Trois (6\%) laissent l'IDE de SSPI informer le patient, 4 (7\%) celle du service. Trois MAR (6\%) laissent le chirurgien l'expliquer. Aucun MAR ne délègue la tâche à son interne. Le contenu de l'information écrite dans le dossier est exposé figure 7. 


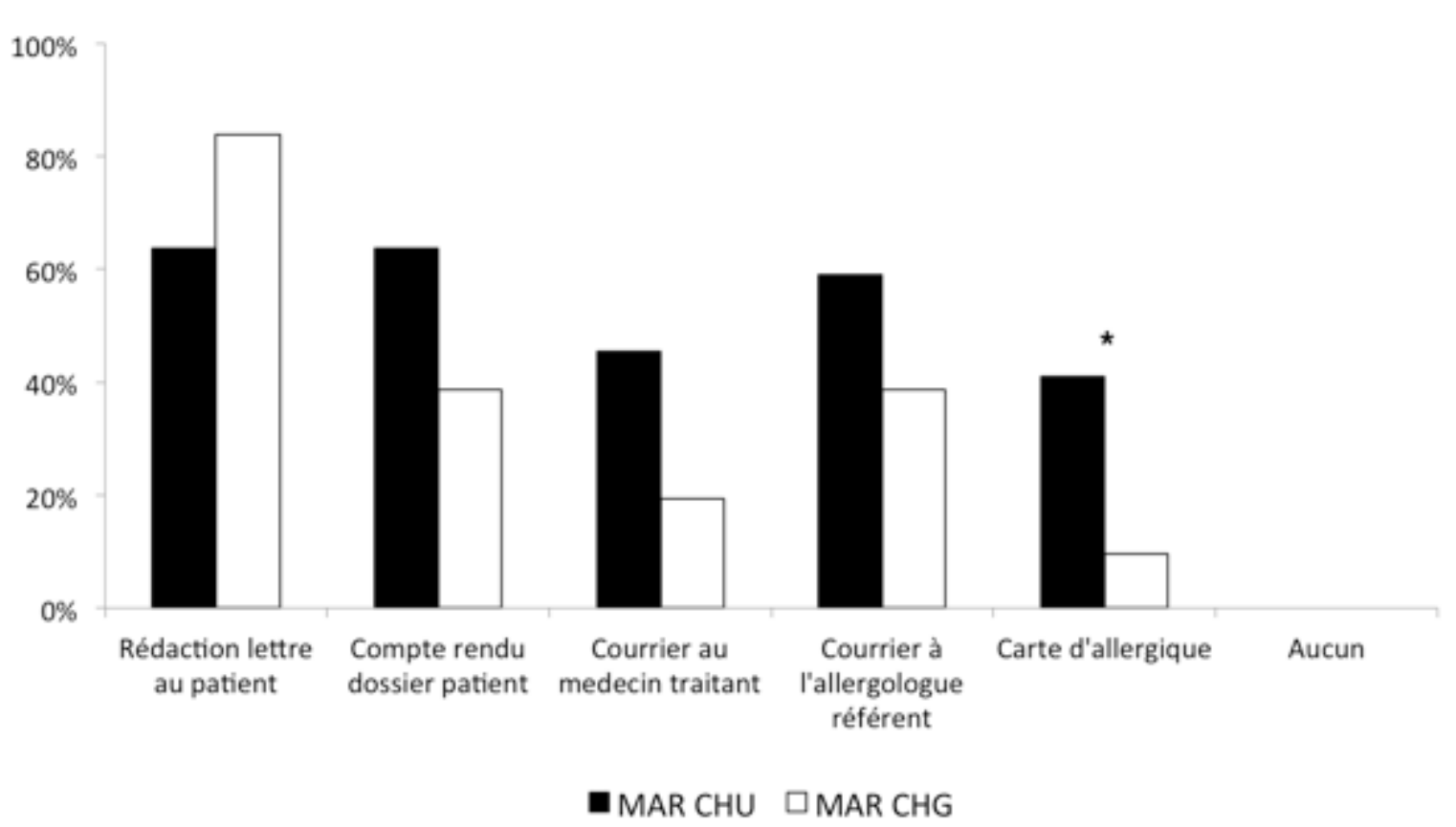

Figure 7 : Description de l'information écrite délivrée au patient. $*=p \leq 0.005$

Quarante-deux (79\%) MAR donnent au patient une lettre personnelle (lettre ou carte d'allergie) à l'issue du CA.

\section{RESULTATS DES BILANS BIOLOGIQUES}

Vingt-trois (43\%) MAR reçoivent les résultats en 10 à 15 jours, 17 (32\%) les reçoivent en un mois et $6(11 \%)$ ne savent pas quand ils doivent les recevoir. Sept (13\%) MAR déclarent recevoir les résultats biologiques dans les 48 heures.

Quarante-six (87\%) MAR reçoivent les résultats le plus souvent par courrier. Dix-huit (34\%) MAR déclarent le plus souvent ne pas recevoir les résultats. La transmission téléphonique est rapportée par cinq MAR (9\%). Aucun ne reçoit les résultats par mail.

Trente-neuf (73\%) MAR savent que ce bilan est insuffisant pour faire le diagnostic.

\section{CONSULTATION D’ALLERGOLOGIE}

Tous les MAR connaissent un allergologue référent qui se trouve au CHU. Quatre (13\%) MAR de CHG ont également un allergologue référent sur leur lieu d'exercice. Les modalités de la prise de rendez-vous et la vérification de la réalisation de la consultation sont détaillées dans les figures 8 et 9. 


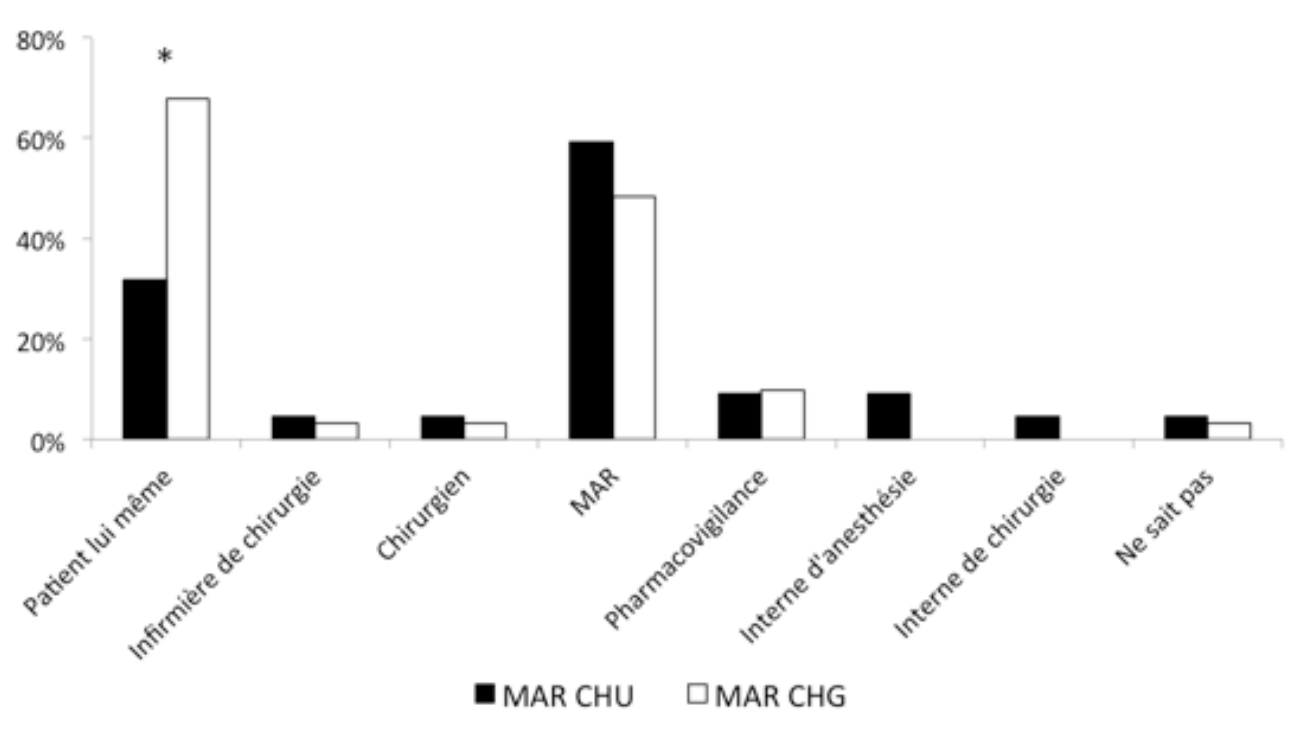

Figure 8 : Modalités de la prise de rendez-vous. $*=p \leq 0.005$

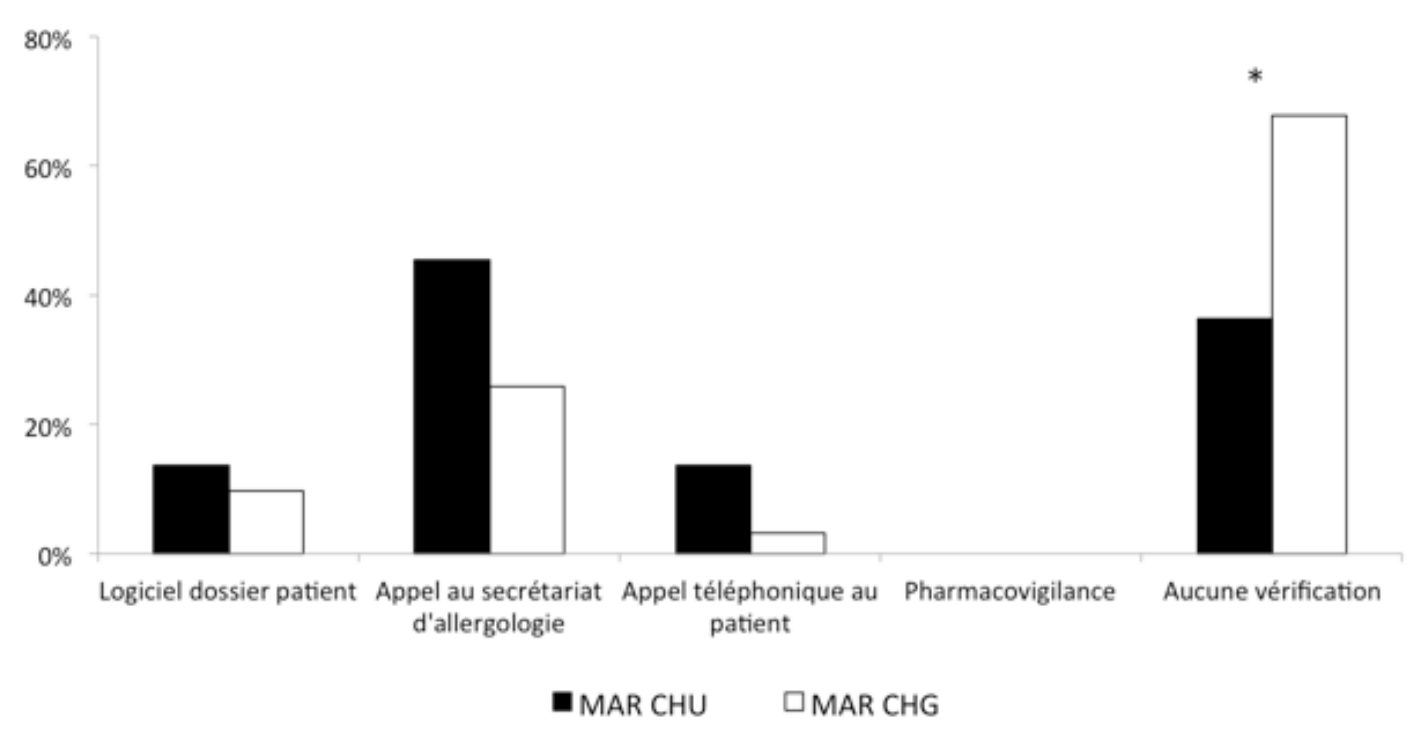

Figure 9 : Modalités de la vérification de la réalisation de la consultation d'allergologie.

$$
*=\mathrm{p} \leq 0.005
$$

Pour trente-neuf (73\%) MAR le délai optimal à partir duquel la consultation d'allergologie est possible est de 4 à 6 semaines après le CA. Pour 11 (21\%), ce délai est de moins d'un mois. Pour 3 (6\%) ce délai est de moins d'une semaine. Aucun ne déclare ignorer les délais.

Les résultats sont obtenus par courrier pour 47 (89\%) MAR. Ils sont reçus par téléphone pour $2(4 \%)$ MAR. Un (3\%) déclare recevoir les résultats par mail. Enfin, 3 (6\%) MAR déclarent ne pas recevoir les résultats. 


\section{CONSULTATION PRE-ANESTHESIQUE D’UN PATIENT ALLERGIQUE}

$\mathrm{Si}$, lors d'une consultation de pré-anesthésie, le patient déclare avoir déjà fait une allergie au décours d'une anesthésie sans pouvoir la préciser, les MAR déclarent rechercher la trace de cette allergie de différentes façons. Celles-ci sont détaillées dans la figure 10.

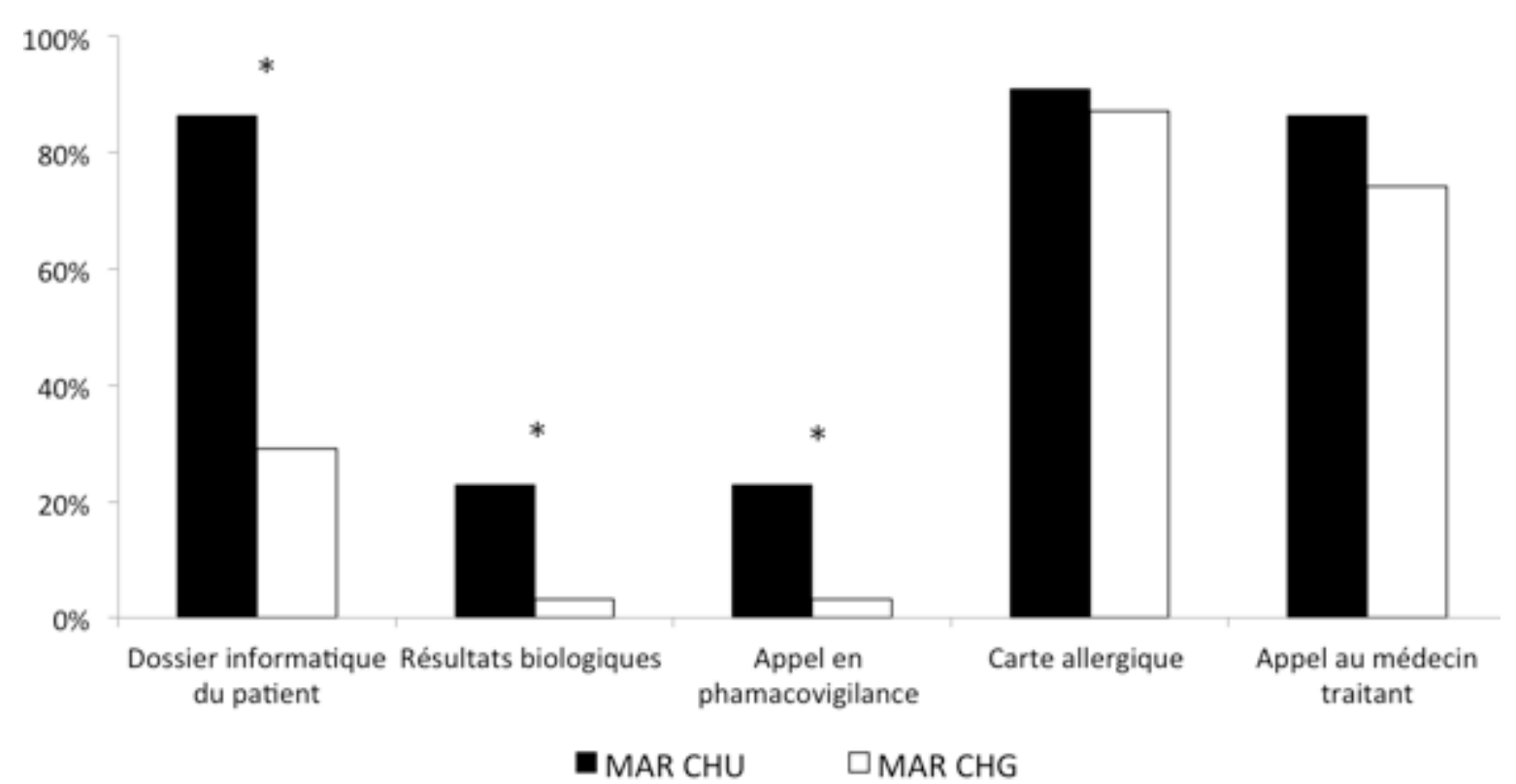

Figure 10 : Modalités de vérification de l'allergie lors d'une CPA. $*=p \leq 0.005$

Si le patient n'a pas bénéficié d'une consultation d'allergologie, trente-quatre (64\%) MAR l'adressent à l'allergologue avant l'intervention, dont 19 (82\%) MAR de CHU et 16 (52\%) MAR de CHG. Si le patient sait décrire son allergie, 24 (45\%) MAR cherchent ses comptes-rendus dans le dossier médical. 


\section{DISCUSSION}

Cette enquête auprès des Anesthésistes de Picardie a permis de mettre en évidence la sous-déclaration des CA auxquels ils étaient confrontés.

Les enquêtes épidémiologiques traitant l'anaphylaxie aux curares sont nombreuses $(4,8,9)$. Cependant les résultats ne sont extrapolés que par rapport aux cas déclarés en pharmacovigilance et aux patients ayant bénéficié d'une consultation d'allergologie. A notre connaissance, aucune étude n'a évalué la réalité de la déclaration en France, et les travaux évaluant la fréquence de la consultation et les moyens entrepris par les MAR pour sa réalisation sont peu nombreux $(18,30)$.

\section{FREQUENCE DES CHOCS ANAPHYLACTIQUES AUX CURARES}

Bien qu'étant la $9^{\text {ème }}$ complication liée à l'anesthésie, la prévalence du choc anaphylactique aux curares est élevée avec un choc pour 5500 anesthésies $(9,11)$. Soixante-huit pour cent des praticiens exerçant en Picardie y ont déjà été confrontés et ce, dès le début de leur exercice professionnel. Cela peut probablement s'expliquer par la fréquence d'utilisation plus élevée des curares et notamment le recours à l'induction par séquence rapide par les plus jeunes. La confrontation des MAR aux chocs de grade 3 ou 4 démontre également qu'il s'agit d'un évènement menaçant le pronostic vital et pour lequel la formation initiale est primordiale en termes de reconnaissance du diagnostic et de prise en charge thérapeutique immédiate. La majorité des MAR ayant eu des CA graves (grade 3-4), ont également eu des chocs moins graves (grade 1-2). Il est probable que ces praticiens soient plus sensibilisés à l'allergie et soient plus vigilants aux réactions cutanées lors de l'injection de curare. Les réactions de grade 1 sont parfois très fugaces et peuvent passer inaperçues. Il est donc possible que la prévalence de l'allergie soit sous-estimée.

\section{INVESTIGATIONS PER ET POST-OPERATOIRES IMMEDIATES}

Le bilan biologique permet de confirmer la réalité de la réaction allergique et d'en identifier les mécanismes. Il comporte 3 dosages. La tryptase, dont le pic sérique est généralement obtenu entre 1 et $2 \mathrm{~h}$ après la réaction, est libérée par les mastocytes activés (31). Son élévation $(\geq 25 \mu \mathrm{g} / \mathrm{L})$ est le témoin d'une réaction anaphylactique IgE-dépendante. Sa 
négativité n'exclue pas le diagnostic. La répétition de son dosage peut être nécessaire pour interpréter des faibles augmentations. L'histamine confirme le mécanisme d'histaminolibération (32). L'élévation est très rapide et le taux sanguin diminue rapidement. Un prélèvement retardé peut méconnaître ce pic. L'association de ces deux dosages augmente la sensibilité diagnostique. Enfin les IgE spécifiques permettent de confirmer le mécanisme immunologique et d'identifier les agents responsables (17). L'horaire du prélèvement n'a pas d'importance. Cependant, réalisé au moment du CA, il permet de prouver la présence d'IgE spécifiques au moment de la réaction. Pour les curares, les IgE spécifiques concernent les ions ammoniums quaternaires. Dans le contexte per-anesthésique, il faut considérer également les autres produits susceptibles d'être à l'origine du choc. Des prélèvements d' IgE anti-hypnotique, anti latex et anti pénicilline peuvent également être indiqués. Cependant la présence d'IgE antiammoniums quaternaires n'implique pas nécessairement la libération d'anticorps lors de l'administration de curares. Ils doivent donc être interprétés en fonction de la clinique et du dosage d'histamine et de tryptase. La combinaison histamine, tryptase et IgE spécifiques possède une sensibilité diagnostique de $87 \%$ et une spécificité de $93.3 \%$ (33).

La grande majorité des MAR fait le dosage de tryptase et d'histamine dans la première heure mais seulement la moitié répète le prélèvement. Les MAR connaissent bien les horaires des prélèvements mais $11 \%$ des patients ne bénéficient pas d'un prélèvement immédiat. L'interprétation à distance par l'allergologue peut être rendue difficile voire impossible. Ceci peut être expliqué par le fait que le patient ne soit plus en danger au moment théorique du $2^{\text {ème }}$ prélèvement et donc que ce prélèvement paraisse moins important aux yeux des MAR. Ces derniers pourraient également remettre en cause leur diagnostic passée la phase d'urgence vitale et décider d'arrêter les investigations. Les grades 1 ou 2 sont d'autant plus complexes à interpréter que les curares peuvent être responsables d'une histaminolibération non allergique. La clinique seule ne permet pas de distinguer l'une et l'autre et c'est pourtant dans ces situations où l'observance du bilan est la moins bonne. Pour les situations les plus graves, les patients sont transférés en réanimation et un défaut de transmission peut aboutir également à un oubli du prélèvement. Enfin, les prélèvements doivent être envoyés individuellement pour permettre une centrifugation rapide. Il peut arriver que les tubes stagnent dans les réfrigérateurs et n'arrivent jamais au laboratoire.

Le dosage des IgE anti-ammoniums quaternaires peut être réalisé durant la phase initiale sans qu'un horaire précis soit nécessaire. Les MAR savent qu'ils doivent les prélever mais il existe une grande variabilité d'horaire pour l'envoi. La demande doit être spécifiquement 
notifiée, car le laboratoire ne rajoute pas un prélèvement qui aurait été oublié. Dix-sept pour cent prélèvent 2 fois les IgE ce qui est inutile, la synthèse de ces anticorps prenant en effet plusieurs jours. Le résultat sera donc le même quel que soit l'horaire du dosage. Cela s'explique car le dosage des IgE est un examen plus spécialisé et peut-être mal maitrisé par les MAR.

Un tiers des MAR ignore quand prélever les leucotriènes urinaires. Ceux-ci ont un rôle dans l'inflammation et entraînent des bronchoconstrictions en réponse à des allergènes. Le dosage des leucotriènes urinaires permet de mettre en évidence une inflammation. Il sont encore régulièrement prélevés, bien que n'apparaissant plus dans les recommandations SFAR (16) ni dans les recommandations anglaises (34). Leur indication reste à évaluer (35).

L'investigation initiale est capitale pour l'orientation du patient. En situation critique, son importance est minimisée par rapport au traitement du patient. La procédure doit être la plus simple possible pour permettre une meilleure observance. Les kits « allergo » doivent être présents en permanence au bloc opératoire (36). Depuis 2007, les kits du CHU ont été améliorés suite aux investigations du médecin allergo-anesthésiste référent (37). Les horaires sont notifiés sur chaque tube sanguin et un flacon de prélèvement urinaire est inclus. Il est important de faire perdurer ces kits pour éviter une baisse de l'observance.

\section{DECLARATION EN PHARMACOVIGILANCE}

Le système national de pharmacovigilance est le plus ancien des systèmes de vigilance sanitaire mis en place en 1973. L'obligation de déclaration est définie comme : « tout médecin, chirurgien-dentiste ou sage-femme ayant constaté un évènement grave ou inattendu susceptible d'être dû à un médicament qu'il l'ait ou non prescrit, doit en faire la déclaration immédiate au centre de pharmacovigilance [...] Tout membre d'une profession de santé ayant fait la constatation peut en informer le centre le pharmacovigilance »(28). Malgré cette obligation légale, seuls $17 \%$ des MAR déclarent leurs chocs tous grades confondus dans notre enquête. Cela est très inférieur au taux national évalué de $45 \%$ dans l'enquête d'impact des recommandations SFAR de Malinovsky (30). Toutefois la question était posée plus directement (déclarez-vous : OUI/ NON) ce qui peut expliquer ce taux de déclaration supérieur. A notre connaissance, il n'y a pas d'études évaluant la déclaration des CA et ceci pour plusieurs raisons : tout d'abord, la déclaration n'apporte pas de bénéfice direct au patient, il s'agit d'un intérêt de santé publique. Les MAR peuvent donc considérer qu'il n'y a pas d'utilité à faire la 
déclaration, privilégiant le prélèvement biologique. De plus la procédure peut être chronophage. Il faut en effet se procurer le formulaire de déclaration de pharmacovigilance, décrire l'incident et faire les copies de l'évènement. Il existe différentes façons de déclarer l'accident (22). Tout d'abord, les kits «allergo » du CHU sont munis d'une feuille de déclaration depuis 2007 pour faciliter cette démarche ce qui augmente le taux de déclaration (37). En l'absence de kit, il est possible de faire la déclaration en ligne par le site internet www.ansn.sante.fr (Agence Nationale de Sécurité du Médicament) (38). La déclaration en ligne n'est toutefois pas toujours disponible suivant le CPR concerné. Il est possible également d'imprimer la feuille de déclaration (39) et on y trouve les coordonnées téléphoniques du CPR. Notre étude montre que les MAR de CHU déclarent quasi exclusivement via la déclaration fournie dans le kit. Les autres moyens de déclaration sont utilisés de façon anecdotique. En revanche, en CHG où ces kits ne sont pas toujours présents, moins de la moitié des MAR les utilisent. La diffusion de ces kits doit se poursuivre pour augmenter l'observance de la déclaration. La déclaration en ligne, qui est une procédure rapide permettant d'éviter toute perte de papier, n'est qu'exceptionnellement utilisée. (40). On peut donc se demander si les MAR connaissent le site de l'ANSN. Ce mode de déclaration a l'avantage d'être disponible quel que soit le lieu d'exercice et l'horaire de survenue du choc. Il évite également au service de pharmacovigilance d'avoir à déchiffrer des feuilles manuscrites.

Par ailleurs, plus de la moitié des MAR envoient les tubes sanguins avec la déclaration. L'ensemble arrive donc au laboratoire de biochimie. Pour permettre de rattraper des déclarations qui n'auraient pas été faites, les agents d'accueil de biochimie du CHU ont été formés à l'envoi des résultats de tryptase et d'histamine au laboratoire de pharmacovigilance (37). Cela permet au service de pharmacovigilance de faire une déclaration spontanée via le laboratoire de biochimie. Le déménagement du CHU, et notamment le regroupement des laboratoires et des secrétariats, a entrainé différents changements de logistique qu'il conviendra d'évaluer. Il est important de poursuivre la formation des agents d'accueil du Centre de Biologie Humaine $(\mathrm{CBH})$ pour assurer l'envoi de la déclaration jointe aux tubes en pharmacovigilance. Cela n'est probablement pas le cas dans tous les hôpitaux généraux.

Le système de déclaration est sensiblement le même en Angleterre via le «Yellow card scheme » mis en place depuis 1964 (12). Toutefois, le site internet est dédié uniquement à la déclaration contrairement au site de l'ANSM et son utilisation est plus intuitive. Au Canada, les praticiens peuvent déclarer leurs évènements indésirables via le «Medeffect Canada ». Il n'y pas de surveillance spécifique aux USA où l'incidence y est plus faible. 
La fréquence des déclarations dépend principalement du grade du choc anaphylactique. Pour les grades 1-2, la sous-déclaration est manifeste. Pourtant $11 \%$ des grades 1 et $22 \%$ des grades 2 sont bel et bien dus à une réaction IgE-dépendante (33). Il est donc difficile d'être sûr qu'un patient n'a jamais présenté de réaction allergique lors de ses précédentes interventions compte tenu de la faible déclaration. Les conséquences pour le patient peuvent être importantes. Il peut ainsi, être réexposé aux curares et présenter un choc plus grave sans que personne ne puisse l'anticiper (41).

Les raisons qui poussent les MAR à ne pas déclarer sont nombreuses: cette démarche semble chronophage, sans bénéfice direct et peut être influencée par un doute diagnostic en l'absence de confirmation immédiate. La crainte d'une sur-déclaration et d'une surcharge de consultations d'allergologie peut freiner le MAR dans cette démarche.

La différence de déclaration selon le grade peut donc expliquer pourquoi les CPR n'ont majoritairement que des déclarations de grades élevés (plus de 70\% de grade 3 et 4) (33). L'épidémiologie actuelle est donc vraisemblablement faussée par cette sous-déclaration.

Pour les cas les plus graves, on pourrait s'attendre à une déclaration systématique. Pourtant 10 à 20\% des MAR ne déclarent «jamais » ou seulement «parfois » les grades 3 ou 4. Dans ces situations, le diagnostic de CA, même si il peut ne pas être le seul envisagé, est plus facile à poser. Nous n'expliquons pas cette absence de déclaration dans les cas sévères. Quoi qu'il en soit, la majorité des MAR ne déclarant pas les cas sévères, ne déclarent en réalité aucun choc.

Après chaque déclaration, une enquête de causalité est réalisée par un médecin de pharmacovigilance et une imputabilité est établie en fonction des critères de Moore (42). Dans le cas des CA suspectés aux curares, la fréquence et le risque de ne pas déclarer est telle que les déclarations sont rarement invalidées par le service de pharmacovigilance. Dans ce travail, rares sont les MAR fournissant la feuille de surveillance per-anesthésique obligeant les médecins de pharmacovigilance à rechercher cette pièce importante du dossier. Lorsque la déclaration est réalisée à distance, le dossier peut être déjà archivé. Cela rend l'analyse du cas difficile $a$ posteriori.

La déclaration doit être faite par tout médecin qui constate la réaction, même si ce n'est pas lui qui a injecté le produit (38). Les patients qui présentent un choc sont amenés à être pris 
en charge par différents intervenants : MAR en salle, MAR en SSPI ou réanimateur. La déclaration pourrait être rattrapée par l'un d'eux.

Dans le logiciel de prescription, il est impossible actuellement de savoir si un patient a fait l'objet d'une déclaration. Une demande est en cours au CHU pour qu'un onglet apparaisse.

\section{INFORMATION DU PATIENT}

Lorsqu'un CA survient, il convient au MAR qui l'a constaté d'informer le patient de la nature de la réaction per-anesthésique et de la nécessité absolue de réaliser un bilan allergologique. La remise d'un courrier détaillé et d'une carte d'allergie provisoire est recommandée (36). L'information doit être orale et écrite. Si la majorité des MAR informe toujours le patient de l'existence d'une allergie et de la nécessité d'en informer leur MAR lors d'une prochaine anesthésie, la moitié seulement les informe « toujours » de la famille de produit suspectée. Peu d'entre eux les informent «toujours » de l'existence d'un prélèvement sanguin et des modalités de la consultation d'allergologie. La majorité des MAR délivre l'information au patient dans le service car la mémorisation du patient en post-opératoire immédiat est médiocre. Mais peu donnent l'information deux fois, en Salle de Surveillance PostInterventionnelle (SSPI) et en service. L'étape de l'information orale est capitale car le patient n'est pas revu à distance par l'équipe d'anesthésie. S'il n'a pas compris l'événement, il sera incapable de le réexpliquer ultérieurement. La mission de l'information au patient dans notre étude est peu déléguée à un autre membre de l'équipe, que ce soit à l'équipe chirurgicale, à l'interne d'anesthésie ou à l'équipe paramédicale, les MAR ayant conscience de leur responsabilité. L'information écrite est majoritairement représentée par une lettre fournie au patient lui-même et rares sont ceux qui fournissent une carte provisoire d'allergie contrairement aux recommandations (16). Ceci peut s'expliquer par l'absence de cartes disponibles aux blocs opératoires. Pourtant, une carte est plus lisible pour le patient, et se range facilement dans un portefeuille, pour être ensuite présentée lors d'une anesthésie ultérieure. Seulement un tiers des MAR informent le médecin traitant. Or, ce dernier recevra les résultats du bilan biologique initial, d'interprétation difficile par l'ignorance des produits injectés et du type de réaction survenue (30). Le risque d'une mauvaise interprétation est d'attribuer à tort la réaction allergique aux curares, ou au contraire de l'exclure alors qu'il s'agit bel et bien d'une réaction allergique. En effet, une étude danoise a montré que l'agent responsable identifié à l'issue des tests cutanés n'est pas toujours celui suspecté par le MAR (43). De plus, si le patient ne sait pas qu'un bilan a été réalisé, il risque de méconnaître l'importance des résultats. 
Un CA est une complication anesthésique qui peut avoir des répercussions médicolégales. Pourtant seule la moitié des MAR laisse une trace écrite dans le dossier. Les logiciels reportant les feuilles de surveillance per-anesthésique se développent dans de nombreux centres mais ne sont pas encore largement diffusés. Il n'est donc pas possible de récupérer ces informations a posteriori si le dossier papier est archivé. De plus, si les lettres fournies au patient sont manuscrites sans copies intégrées dans le dossier, la preuve de la délivrance de l'information ne pourra pas être faite. Cela peut s'expliquer par plusieurs raisons. Tout d'abord, l'utilisation du logiciel informatique pour rédiger des comptes rendus est peu répandue en anesthésie. De plus, il existe un manque d'anesthésistes dans de nombreux centres (44). La répartition du temps de travail entre le bloc et le service est majoritairement tournée vers le bloc opératoire. Le manque de temps oblige à aller à l'essentiel. Dans le cas des CA, les MAR priorisent le traitement urgent. L'information donnée au patient est bonne puisqu'une lettre personnelle est fournie dans la majorité des cas. Toutefois, cette information n'est donnée que si le MAR a bien retenu le diagnostic.

\section{RESULTATS DES INVESTIGATIONS IMMEDIATES}

L'analyse des prélèvements allergiques n'est pas actuellement disponible en Picardie. Ils sont généralement envoyés au laboratoire CERBA. Les tubes transitent par le laboratoire de biochimie pour permettre la centrifugation. La multiplication des intervenants augmente le risque de perte de tubes et les délais d'obtention des résultats. Ceci explique pourquoi les MAR reçoivent les résultats à des délais variables et que certains ignorent le délai de réception de ces résultats.

Les résultats arrivent au laboratoire d'immunologie du CHU d'Amiens puis sont envoyés au MAR déclarant, au patient et au médecin traitant sous forme papier. La majorité des MAR récupère les résultats par courrier mais un tiers déclare ne pas les recevoir. Cela concorde avec l'étude de Malinovski et coll. où 38\% des anesthésistes déclarent avoir des difficultés à obtenir les résultats du bilan (30). Lors de cette étape, il est possible de rattraper une non-déclaration et une mauvaise orientation du patient, puisqu'au CHU, les résultats sont également envoyés en pharmacovigilance et à la consultation d'allergologie. Si le nom du MAR déclarant n'était pas lisible ou non renseigné, ces résultats bien qu'envoyés dans le département d'anesthésie, risquent d'être perdus. 
Les résultats ne sont pas encore rapportés dans le dossier informatique du patient. Si le courrier est perdu, pour les obtenir, il faut appeler au laboratoire extérieur ce qui est fastidieux. Une demande est en cours pour que ces résultats soient accessibles.

Enfin, il faut préciser que les prélèvements analysés en laboratoire privé impliquent des coûts financiers pris en charge par les $\mathrm{CH}$ publics, mais dans les établissements de santé privés, les patients doivent avancer les frais pour obtenir les résultats. Cela peut entraîner des incompréhensions de leur part et parfois même un refus de payer, surtout si il n'a pas été informé de l'existence de ce prélèvement.

\section{CONSULTATION D’ALLERGOLOGIE}

La consultation d'allergologie peut être réalisée à partir de 4 à 6 semaines de façon préférentielle. Elle peut être effectuée plus précocement en cas d'urgence mais seuls les résultats positifs sont valables (31). Il existe en France 40 centres spécialisés en allergoanesthésie géré par le Groupe d'Etude des Réactions Anaphylactoïdes Per-anesthésiques (GERAP). Diriger les patients vers ces centres est préférable car le diagnostic de l'allergie et surtout la confirmation de l'agent responsable peut être difficile par un allergologue non spécialisé (10). Il est recommandé de tester les allergies croisées aux autres curares, mais seulement la moitié des allergologues généralistes les testent, d'où l'importance d'adresser le patient au centre référent (30). De plus, la surveillance des patients après les tests est renforcée et implique la présence de matériel et d'unestructure de réanimation. La majorité des MAR connaît le centre de référence qui est en Picardie au CHU d'Amiens. Sur le plan national, seulement 50\% des MAR connaissent le site référent (30). Toutefois, les délais de consultation peuvent être de plusieurs mois en raison du nombre de patients à tester par allergologue. Les coordonnées des différents centres GERAP sont difficilement accessibles et il peut être difficile d'adresser le patient dans un autre centre si le délai est trop long. A l'étranger, il existe également des centres d'allergologie de référence mais ils sont peu nombreux : un seul centre réfèrent au Danemark et quatre en Norvège (45).

La moitié des MAR prennent eux même le rendez-vous d'allergologie, tandis que l'autre moitié laisse le patient le faire. Les coordonnées des allergologues référents n'étant pas clairement visibles sur internet ou sur les pages jaunes, le patient peut se retrouver en difficulté pour prendre ce rendez-vous a posteriori. De plus, seulement la moitié des MAR vérifient que le patient a bien consulté. Ceci contribue à la faible part des patients qui vont au bout de la démarche diagnostique. 
La grande majorité des MAR reçoit bien les résultats de la consultation d'allergologie, objectivant des liens MAR-allergologues plus importants que sur le plan national (30). Cette consultation permet de faire le diagnostic positif et surtout de diagnostiquer les allergies croisées. Pourtant pour $26 \%$ des MAR, le bilan biologique suffit. Il y a donc un manque d'information sur ce point.

De plus, bien que n'ayant pas cette vocation, le centre de pharmacovigilance lorsqu'il a connaissance d'un bilan en faveur d'une réaction IgE médiée rappelle souvent les MAR pour savoir si la consultation d'allergologie a bien eu lieu.

\section{CONSULTATION PRE-ANESTHESIQUE DU PATIENT ALLERGIQUE}

Lorsque le MAR voit en consultation pré-anesthésique un patient qui se dit allergique, il peut être difficile de retrouver les informations nécessaires même si celui-ci a été anesthésié dans le même centre. Si la majorité des MAR contacte le médecin traitant pour obtenir cette information, ils n'étaient que la moitié à l'informer. De la même façon, une majorité demande la carte allergique du patient alors qu'ils étaient peu à en fournir. Les MAR comptent certainement sur l'allergologue pour fournir cette carte définitive. Pourtant seuls $60 \%$ des patients ? bénéficient de cette consultation (5).

Au CHU, une majorité consulte le dossier informatique du patient. Néanmoins, les feuilles de surveillance per-anesthésiques n'y figurent que depuis peu. Même si seulement la moitié des patients aura une notification faite par leur MAR déclarant, il est également possible de trouver des renseignements dans le compte-rendu chirurgical ou encore fréquemment dans les transmissions paramédicales.

Une minorité des MAR contacte le centre de pharmacovigilance. Toutefois, si la déclaration a été faite, il est possible de ressortir rapidement un dossier récent. Pour les dossiers anciens, une fiche anonyme avec un code ANSM informatisée peut être récupérée sans délai. Y sont regroupées l'ensemble des informations importantes : produits injectés, grade de réaction, résultats biologique et consultation d'allergologie parfois même si elle a été effectuée dans un autre établissement. Cela met en évidence le manque de communication entre les MAR et la pharmacovigilance.

Lorsque le patient allergique n'a pas bénéficié d'une consultation, la majorité des MAR du CHU l'adresse avant l'intervention mais seulement la moitié des MAR de CHG. Les délais de ces consultations et leur distance par rapport au domicile du patient expliquent peut-être 
cette différence. Les sites de consultations, bien que nettement plus nombreux qu'à l'étranger, restent peu développés à l'échelle d'une région $(27,30)$. Si les liens entre la consultation d'allergologie et les MAR sont bien développés au niveau du CHU, ils doivent encore être améliorés sur le plan régional (46).

\section{$\underline{\text { AMELIORATIONS ET PERSPECTIVES }}$}

La diffusion des kits «allergo » doit être poursuivie au CHU et dans les CHG. Ils permettent de rappeler les horaires des prélèvements et de ne pas avoir à chercher des bons de demande d'examens extérieurs. La feuille de déclaration au CPR est toujours présente dans ce kit au CHU ce qui n'est pas le cas dans l'ensemble des CHG. Cette procédure doit être étendue à l'ensemble des hôpitaux pour permettre une plus grande diffusion. La diffusion du site internet de l'ANSM doit être encouragée et si possible notifiée dans les protocoles d'urgences vitales affichés dans les blocs opératoires. De plus, une configuration du site sur le modèle anglais permettrait d'augmenter et faciliter les demandes en ligne.

\section{$\underline{\text { LIMITES }}$}

Il s'agit d'une étude régionale portant sur un effectif limité. Les résultats sont à interpréter avec prudence sur le plan national. En effet, il peut exister des différences locales sur l'envoi de la déclaration et sur l'organisation de la consultation d'allergologie. Les praticiens interrogés travaillaient uniquement en secteur hospitalier. Toutefois, $30 \%$ des MAR travaillent en France en secteur libéral exclusif (47). Les cas de déclarations spontanées venant d'établissements privés sont minoritaires, la sous déclaration pourrait donc être encore plus importante. Le taux de participation est de 64\%, mais les médecins sont fréquemment sollicités pour répondre à des questionnaires, impliquant une certaine lassitude. De plus, certains peuvent ne pas se sentir impliqués dans cette enquête s'ils n'ont jamais été confrontés à un CA ou s'ils ignorent les missions de la pharmacovigilance. Cela reste un taux de réponse satisfaisant pour ce type d'étude,

Le caractère déclaratif de ce travail comporte d'autres limites: l'ordre choisi pour les questions peut induire un biais de halo et d'ancrage, pouvant se réduire par la présentation aléatoire des questions mais rendant la participation fastidieuse. Le biais de désirabilité sociale incitant la personne sondée à orienter ses réponses positivement aux yeux de l'enquêteur est également limitant dans cette étude. 


\section{CONCLUSION}

Ce travail a permis de démontrer que les MAR étaient fréquemment confrontés au CA.

La sous-déclaration supposée de ces évènements a été confirmée, plus particulièrement lors des grades faibles mais la déclaration des grades élevés n'est pour autant pas systématique.

La présence de feuille de déclaration des CA doit être poursuivie au CHU et encouragée dans les CHG. De même, la diffusion du site internet de pharmacovigilance doit être encouragée dans l'ensemble des hôpitaux comme moyen alternatif en cas de non disponibilité de ces kits.

Les investigations immédiates sont correctement effectuées pour la $1^{\text {ère }}$ heure mais l'observance diminue par la suite. La présence des kits « allergo » est donc primordiale pour rappeler aux MAR l'horaire et le type de prélèvement.

Les liens entre la consultation d'allergologie et les MAR sont forts sur le plan régional, mais la distance et les délais peuvent être des freins.

L'information des patients par le MAR déclarant doit être développée notamment sur les enjeux de la consultation d'allergologie. La carte d'allergique pourrait être matériellement représentée telle une «carte vitale », facilement rangée dans un portefeuille. Une consultation intermédiaire à distance du choc et avant la consultation d'allergologie pourrait être proposée pour réexpliquer la procédure au patient et diminuer le nombre de patients non diagnostiqués. 


\section{BIBLIOGRAPHIE}

1. Weiser TG, Regenbogen SE, Thompson KD, Haynes AB, Lipsitz SR, Berry WR, et al. An estimation of the global volume of surgery: a modelling strategy based on available data. Lancet. 2008 Jul 12;372(9633):139-44.

2. Pearse RM, Bauer P, Pelosi P, Metnitz P, Spies C. Mortality after surgery in Europe: a 7 day cohort study. 80(9847). 2012 Sep 22;380(9847:1059-65.

3. Hatton F, Tiret L, Maujol L, N'Doye P, Vourc'h G, Desmonts JM, et al. [INSERM. Epidemiological survey of anesthesia. Initial results]. Ann Fr Anesthèsie Rèanimation. 1983;2(5):331-86.

4. Mertes PM, Alla F, Tréchot P, Auroy Y, Jougla E, Groupe d'Etudes des Réactions Anaphylactoïdes Peranesthésiques. Anaphylaxis during anesthesia in France: an 8-year national survey. J Allergy Clin Immunol. 2011 Aug;128(2):366-73.

5. Mertes PM, Petitpain N, Malinovsky J-M, Gillet P. Allergie et curares : évolution de l'épidémiologie (podcast). Prat En Anesth Réanimation. 2014 Jun;18(3):158-63.

6. Watkins J. Adverse anaesthetic reactions. An update from a proposed national reporting and advisory service. Anaesthesia. 1985 Aug;40(8):797-800.

7. Harboe T, Guttormsen AB, Irgens A, Dybendal T, Florvaag E. Anaphylaxis during anesthesia in Norway: a 6-year single-center follow-up study. Anesthesiology. 2005 May;102(5):897-903.

8. Laxenaire MC, Mertes PM, Groupe d'Etudes des Réactions Anaphylactoïdes Peranesthésiques. Anaphylaxis during anaesthesia. Results of a two-year survey in France. Br J Anaesth. 2001 Oct;87(4):549-58.

9. Mertes P-M, Laxenaire M-C. Épidémiologie des réactions anaphylactiques et anaphylactoïdes peranesthésiques en France. Septième enquête multicentrique (Janvier 2001-Décembre 2002). Ann Fr Anesth Réanimation. 2004 Dec;23(12):1133-43.

10. Laxenaire MC. Quelle est la réalité du risque allergique en anesthésie ? Incidence. Aspects cliniques. Morbidité-mortalité. Substances responsables. /data/revues/18770320/v0042i05/02001880/ [Internet]. [cited 2015 Jan 26]; Available from: http://www.em-consulte.com/en/article/10980

11. Mertes PM, Malinovsky JM, Jouffroy L, Working Group of the SFAR and SFA, Aberer W, Terreehorst I, et al. Reducing the risk of anaphylaxis during anesthesia: 2011 updated guidelines for clinical practice. J Investig Allergol Clin Immunol. 2011;21(6):442-53.

12. Light KP, Lovell AT, Butt H, Fauvel NJ, Holdcroft A. Adverse effects of neuromuscular blocking agents based on yellow card reporting in the U.K.: are there differences between males and females? Pharmacoepidemiol Drug Saf. 2006 Mar;15(3):151-60.

13. Fisher MM, Baldo BA. The incidence and clinical features of anaphylactic reactions during anesthesia in Australia. Ann Fr Anesthèsie Rèanimation. 1993;12(2):97-104. 
14. Reitter M, Petitpain N, Latarche C, Gillet P, Audibert G, Mertes PM. Décès par choc anaphylactique aux curares: analyse des facteurs de risque et de la prise en charge therapeutique. Ann Fr Anesth Réanimation. 2014 Sep;33, Supplement 2:A98.

15. Newland MC, Ellis SJ, Lydiatt CA, Peters KR, Tinker JH, Romberger DJ, et al. Anestheticrelated cardiac arrest and its mortality: a report covering 72,959 anesthetics over 10 years from a US teaching hospital. Anesthesiology. 2002 Jul;97(1):108-15.

16. SFAR. Prévention du risque allergique peranesthésique. 2001.

17. Guilloux L, Benoit Y, Aimone-Gastin I, Ponvert C, Beaudouin E. Conduite du bilan diagnostique biologique. Les immunoglobulines E. Ann Fr Anesth Réanimation. 2011 Mar;30(3):294-304.

18. Moneret-Vautrin DA. Test cutanés pour le diagnostic d'allergie aux curares. Ann Fr Anesth Réanimation. 2002 May;21, Supplement 1:97-107.

19. Lucas-Polomeni M-M, Rochefort-Morel C, Delaval Y. Bilan allergologique lors de la consultation de préanesthésie : pour qui ? Comment ? Prat En Anesth Réanimation. 2008 Jun;12(3):158-66.

20. BREUIL K, REMBLIER C, MEURICE JC. intérêt d'une consultation d'allergologie à la suite d'un accident peranesthésique - bilan d'un an d'actvité au chu de poitiers. 2000.

21. Dewachter P, Mouton-Faivre C, Pertek J-P, Boudaa C, Mertes P-M. Intérêt des tests cutanés pour le choix d'un curare après une réaction anaphylactique. Ann Fr Anesth Réanimation. 2005 May;24(5):543-6.

22. http://ansm.sante.fr/Declarer-un-effet-indesirable/Pharmacovigilance/Centres-regionauxde-pharmacovigilance/(offset)/4.

23. A. Spreux BB. La pharmacovigilance en pratique. Transfus Clin Biol - TRANSFUS CLIN Biol. 1999;6(4):254-9.

24. La pharmacovigilance I. Structure et activités du système français [Internet]. EM-Consulte. [cited 2015 Mar 5]. Available from: http://www.em-consulte.com/it/article/191760/lapharmacovigilance-i-structure-et-activites-du-s

25. ANSM. Comité technique de pharmacovilance du 21 mai 2013. Available from: http://ansm.sante.fr/var/ansm_site/storage/original/application/47860dababd65356aee23a 7c82974954.pdf

26. Petitpain N, Mertes P-M, Malinovsky JM, Drouet M, Gillet P. ALPHO : ALlergy to PHOlcdine and NBMBA anaphylaxis.

27. Laroche D, Bricard H, Laxenaire MC. Consultation d'allergoanesthesie : pas assez de patients testes apres un accident anaphylactoide peranesthesique. Ann Fr Anesth Reanim. 1998;1(17):89-90.

28. JORF. Décret. Décret n84-402 mai, 1984 p. 1686. 
29. Ring J, Messmer K. Incidence and severity of anaphylactoid reactions to colloid volume substitutes. Lancet. 1977 Feb 26;1(8009):466-9.

30. Malinovsky J-M, Demoly P, Lavaud F, Mertes P-M. Enquêtes d'impact des recommandations Sfar-SFA de 2002 sur la «Prévention du risque allergique peranesthésique ». Ann Fr Anesth Réanimation. 2011 Mar;30(3):207-11.

31. Laroche D, Lefrançois C, Gérard JL, Dubois F, Vergnaud MC, Guéant JL, et al. Early diagnosis of anaphylactic reactions to neuromuscular blocking drugs. Br J Anaesth. 1992 Dec;69(6):611-4.

32. Laroche D, Dubois F, Gérard JL, Lefrançois C, André B, Vergnaud MC, et al. Radioimmunoassay for plasma histamine: a study of false positive and false negative values. Br J Anaesth. 1995 Apr;74(4):430-7.

33. Mertes P-M, Malinovsky J-M, Alla F, Studnicska D, Tréchot P, Laxenaire M-C. Épidémiologie des réactions d'hypersensibilité immédiates peranesthésiques chez l'adulte et l'enfant: Bilan de huit années d'enquête du Gerap. Rev Fr Allergol Immunol Clin. 2008 Apr;48(3):217-21.

34. Ewan PW, Dugué P, Mirakian R, Dixon TA, Harper JN, Nasser SM, et al. BSACI guidelines for the investigation of suspected anaphylaxis during general anaesthesia. Clin Exp Allergy J Br Soc Allergy Clin Immunol. 2010 Jan;40(1):15-31.

35. Sabbah A, Drouet M, el Founini M. [Importance of the urinary leukotriene E4 level. Preliminary study]. Allerg Immunol (Leipz). 1998 Jun;30(6):177-8.

36. Mertes PM, Malinovsky JM, Jouffroy L, Working Group of the SFAR and SFA, Aberer W, Terreehorst I, et al. Reducing the risk of anaphylaxis during anesthesia: 2011 updated guidelines for clinical practice. J Investig Allergol Clin Immunol. 2011;21(6):442-53.

37. Perquin M. Analyse des différentes étapes de l'enquête allergologique lors d'une suspicion de réaction d'hypersensibilité immédiate peropératoire au CHU d'AMIENS de 2008 à 2010 : conduite des investigations allergologiques et propositions d'amélioration. SFAR $2011 ; 2011$.

38. http://ansm.sante.fr/Declarer-un-effet-indesirable/Votre-declaration-concerne-unmedicament/Votre-declaration-concerne-un-medicament/(offset)/ 0 .

39. https://www.formulaires.modernisation.gouv.fr/gf/cerfa_10011.do.

40. Micoulaud-Franchi J-A. Un pas de plus vers une pharmacovigilance 2.0: Intégration des données du web communautaire à une pharmacovigilance plus alerte. Presse Médicale. 2011;40(9):790-2.

41. Pedersen AF, Green S, Rose MA. Failure to investigate anaesthetic anaphylaxis resulting in a preventable second anaphylactic reaction. Anaesth Intensive Care. 2012 Nov;40(6):1053-5.

42. Moore N, Biour M, Paux G, Loupi E, Begaud B, Boismare F, et al. Adverse drug reaction monitoring: doing it the French way. Lancet. 1985 Nov 9;2(8463):1056-8. 
43. Krøigaard M, Garvey LH, Menné T, Husum B. Allergic reactions in anaesthesia: are suspected causes confirmed on subsequent testing? Br J Anaesth. 2005 Oct;95(4):468-71.

44. Auboyer C. La démographie des anesthésistes-réanimateurs en 2020 : un problème ou une catastrophe ? Ann Fr Anesth Réanimation. 2010 Dec;29(12):857-8.

45. Kroigaard M, Garvey LH, Gillberg L, Johansson SGO, Mosbech H, Florvaag E, et al. Scandinavian Clinical Practice Guidelines on the diagnosis, management and follow-up of anaphylaxis during anaesthesia. Acta Anaesthesiol Scand. 2007 Jul;51(6):655-70.

46. Nicolie B, Callandre E, Cottineau C, Beydon L. Les frontières du bilan allergologique en allergo-anesthésie. Rev Fr Allergol Immunol Clin. 2008 Dec;48(8):543-7.

47. Pontone S, Brouard N, Scherpereel P, Boulard G, Arduin P. Les médecins anesthésistesréanimateurs en France en 1999 Premiers résultats de l'enquête démographique nationale Cfar-Sfar-Ined. Ann Fr Anesth Réanimation. 2002 Dec;21(10):779-806. 


\section{ANNEXE 1: Prise en charge d'un choc anaphylactique}

SIGNES CLINIQUES

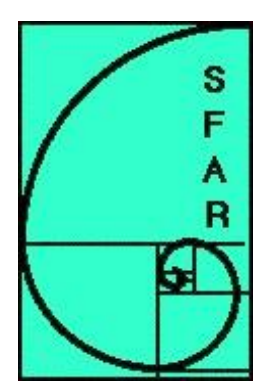

spécifique.

GRADE I Signes cutanéomuqueux généralisés.

GRADE II Atteinte multiviscérale modérée (au moins 2 fonctions atteintes)

GRADE III Atteinte multiviscérale sévère menaçant la vie et imposant un traitement

GRADE IV Les signes cutanés peuvent être absents ou n'apparaître qu'après la remontée tensionnelle Arrêt circulatoire et/ou respiratoires . www.sfar.org

\section{TRAITEMENT}

- Appel aide urgente, et arrêt de l'injection du produit suspecté si possible

- Concertation médico-chirurgicale (abstention, simplification, accélération ou arrêt du geste chirurgical)

- Oxygène pur et contrôle rapide des voies aériennes • Voie veineuse efficace

REMPLISSAGE VASCULAIRE : cristalloïdes isotoniques $\left(30 \mathrm{~mL} \cdot \mathrm{kg}_{-1}\right)$ puis amidons (30 $\left.\mathrm{mL} \cdot \mathrm{kg}_{-1}\right)$

ADRENALINE IV par titration, toutes les 1 à 2 min, selon le grade de sévérité de la réaction

La tachycardie ne contre-indique pas l'utilisation d'adrénaline

- Grade I : pas d'adrénaline

- Grade II : bolus de 10 à $20 \mu \mathrm{g}$

- Grade III : bolus de 100 à $200 \mu \mathrm{g}$

- Grade IV : traitement d'un arrêt circulatoire - MCE : massage cardiaque externe

- BOLUS de $1 \mathrm{mg}$ d'ADRENALINE toutes les 1 à 2 minutes puis $5 \mathrm{mg}$ à partir de la 3 ìme injection,

Les doses d'adrénaline doivent être augmentées, puis relayées par une perfusion continue de 0,05 à $0,1 \mu \mathrm{g} \cdot \mathrm{kg}^{-1} \cdot \mathrm{min}^{-1}$.

\section{CAS PARTICULIERS}

\section{BRONCHOSPASME}

salbutamol spray (Ventoline ${ }^{\circledR}$ ) ou si forme d'emblée sévère salbutamol IV (Salbumol ${ }^{\circledR}$ )

- $\quad$ bolus 100 et $200 \mu \mathrm{g}$ en perfusion continue (5 à $25 \mu \mathrm{g} \cdot \min _{-1}$ ) adrénaline en perfusion continue dans les formes les plus graves les corticoïdes ne représentent pas le traitement de première intention

\section{FEMME ENCEINTE}

Décubitus latéral gauche

Remplissage vasculaire : cristalloïdes puis amidons (comme chez une femme non enceinte)

ADRENALINE

- $\quad 1^{\text {er }}$ bolus de 100 à $200 \mu \mathrm{g}$ IVD,

- $\quad$ renouvelé toutes les 1 à 2 minutes selon l'effet obtenu

\section{ENFANT}

Remplissage vasculaire : cristalloïdes $\left(20 \mathrm{ml} . \mathrm{kg}^{-1}\right)$ puis colloïdes $\left(10 \mathrm{ml} \cdot \mathrm{kg}^{-1}\right)$.

ADRENALINE : 
- $\quad \mathbf{1}^{\text {er }}$ bolus de $1 \mu \mathrm{g} . \mathrm{kg}^{-1}$, jusqu'à 5 à $10 \mu \mathrm{g} . \mathrm{kg}^{-1}$

- $\quad$ En cas d'arrêt circulatoire (grade IV), bolus de $10 \mu \mathrm{g} . \mathrm{kg}^{-1}$.

- Les bolus itératifs d'adrénaline peuvent être relayés par une perfusion continue débutée à $0,1 \mu \mathrm{g} \cdot \mathrm{kg}^{-1} \cdot \mathrm{min}^{-1}$.

\section{PATIENT TRAITE PAR B-BLOQUANTS}

Augmenter la posologie d'adrénaline

En cas d'inefficacité de l'adrénaline : glucagon (1 à 2 mg IVD) à renouveler toutes les 5 minutes

COLLAPSUS CARDIOVASCULAIRE REFRACTAIRE A L'ADRENALINE (>10 mg injectés)

Noradrénaline : $0,1 \mu \mathrm{g} \cdot \mathrm{kg}^{-1} \cdot \mathrm{min}^{-1}$

INVESTIGATIONS ALLERGOLOGIQUES

Prélèvements immédiats

\section{Tests cutanés à distance}

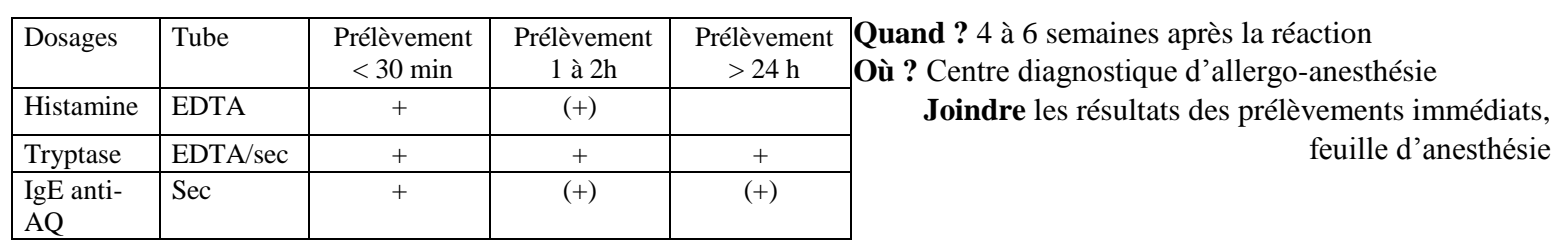

Déclaration au centre régional de pharmacovigilance, en utilisant e formulaire disponible sur le site 


\section{ANNEXE 2: Questionnaire}

Evaluation de la déclaration de pharmacovigilance des chocs anaphylactiques aux curares *Obligatoire

Evaluation de la déclaration de pharmacovigilance des chocs anaphylactiques aux curares

4/A combien de chocs anaphylactiques aux curares avez-vous déjà été confronté en peropératoire?

Les manifestations cliniques sont décrites suivant quatre stades de gravité croissante : 1 : signes cutanéomuqueux genéralises : erytheme, urticaire, avec ou sans œedeme angioneurotique II : atteint multiviscerale modéree, avec signes cutaneo-muqueux, hypotension et tachycardie inhabituelles, hyperreactivité bronchique (toux, difficulté ventilatoire) III : atteinte multiviscerale severe menaçant la imposant une therapeutique spécifique = collapsus, tachycardie ou bradycardie, troubles du rythme cardiaque, bronchospasme, les signes cutanés peuvent être absents ou n'apparaître qu'après la remo tensionnelle. IV : arrêt circulatoire et/ou res

\begin{tabular}{cccc} 
& 0 & $\leq 5$ & $>5$ \\
\hline Grade 1 & 0 & 0 & 0 \\
\hline Grade 2 & 0 & 0 & $\bigcirc$ \\
\hline Grade 3 & 0 & 0 & 0 \\
\hline Grade 4 & 0 & 0 & $\bigcirc$
\end{tabular}

5/Lorsque vous y êtes confrontés, à quelle fréquence réalisez vous cette déclaration?

\begin{tabular}{|c|c|c|c|c|}
\hline & Jamais & Parfois & Souvent & Toujours \\
\hline Grade 1 & 0 & 0 & $\bigcirc$ & 0 \\
\hline Grade 2 & 0 & $\odot$ & $\bigcirc$ & 0 \\
\hline Grade 3 & 0 & 0 & 0 & 0 \\
\hline Grade 4 & 0 & 0 & 0 & 0 \\
\hline
\end{tabular}

6 A/Quelles sont les horaires des prélèvements pharmacologiques: pour l'histamine? *
$\square$ HO-H1
$\square \mathrm{H} 1-\mathrm{H} 2$
$\square$ н3
$\square \mathrm{H} 12-\mathrm{H} 24$
$\square$ ne sait pas

$6 \mathrm{~B} /$ Quelles sont les horaires des prélèvements pharmacologiques: pour la tryptase? *

$\square$ HO-H1

$\square \mathrm{H} 1-\mathrm{H} 2$

$\square \mathrm{H3}$

$\square \mathrm{H} 12-\mathrm{H} 24$

$\square$ ne sait pas

$6 \mathrm{C} /$ Quelles sont les horaires des prélèvements pharmacologiques: pour les Ige spécifiques?

$\square \mathrm{HO}-\mathrm{H} 1$

$\square \mathrm{H} 1-\mathrm{H} 2$

$\square \mathrm{H3}$

$\square \mathrm{H} 12-\mathrm{H} 24$

$\square$ ne sait pas

6 D/Quelles sont les horaires des prélèvements pharmacologiques: pour les leucotriènes urinaires? *

$\square \mathrm{HO}-\mathrm{H} 1$

$\square \mathrm{H} 1-\mathrm{H} 2$

$\square$ н3

$\square \mathrm{H} 12-\mathrm{H} 24$

$\square$ ne sait pas 
7/Qui s'occupe de l'envoi des prélèvements au laboratoire? *

$\square$ IADE de salle d'intervention

$\square$ interne d'anesthésie

$\square$ Infirmière de service de chirurgie

$\square$ vous même

Infirmière ou IADE de SSPI

$\square$ ne sait pas

8/Comment réalisez-vous la déclaration d'allergie? *

Formulaire de déclaration de pharmacovigilance présent dans le kit choc anaphylactique dédié

Appel téléphonique au centre de pharmacovigilance

Envoi d'un mail au centre de pharmacovigilance

Déclaration en ligne

Envoi d'un courrier

Ne sait pas

9/Quelles pièces fournissez-vous lors de la déclaration? *

Feuille de déclaration présente dans un kit "choc" dédie

$\square$ Description manuscrite de l'évènement

$\square$ copie de la consultation pré anesthésique

$\square$ copie de la feuille de surveillance d'anesthésie

$\checkmark$ les tubes sanguins

$\square$ ne sait pas

10/Comment informez-vous le patient? *

en sspi: information donnée par vous même

$\square$ en sspi: information donnée par l'infirmière

en service: information donnée par vous même

en service: information donnée par l'infirmière

en service: information donnée par le chirurgien

$\square$ information donnée par l'interne d'anesthésie

11/Quelle information donnez-vous au patient?

\begin{tabular}{|c|c|c|c|c|}
\hline & Jamais & Parfois & Souvent & Toujours \\
\hline $\begin{array}{l}\text { Existence d'une } \\
\text { allergie per- } \\
\text { opératoire }\end{array}$ & 0 & 0 & 0 & 0 \\
\hline $\begin{array}{l}\text { La famille de } \\
\text { produit suspectée }\end{array}$ & 0 & 0 & 0 & 0 \\
\hline $\begin{array}{l}\text { Prélèvement } \\
\text { sanguin per- } \\
\text { opératoire }\end{array}$ & 0 & 0 & 0 & 0 \\
\hline $\begin{array}{l}\text { Nécessité d'en } \\
\text { informer } \\
\text { l'anesthésiste lors } \\
\text { d'une prochaine } \\
\text { intervention }\end{array}$ & 0 & 0 & 0 & 0 \\
\hline $\begin{array}{l}\text { Modalités } \\
\text { d'obtention des } \\
\text { résultats du } \\
\text { prélèverment } \\
\text { sanguin }\end{array}$ & 0 & 0 & 0 & 0 \\
\hline $\begin{array}{l}\text { Modalités de } \\
\text { réalisation du } \\
\text { bilan } \\
\text { allergologique }\end{array}$ & 0 & 0 & 0 & 0 \\
\hline
\end{tabular}

12/Laissez-vous une trace écrite de la réaction allergique et de la déclaration? *

$\square$ Lettre remise en mains propres au patient

Compte rendu dans un logiciel de dossier patient ou manuscrit dans un dossier papier

Courrier au médecin traitant

$\checkmark$ Courrier pour l'allergologue

carte d'allergique

$\square$ aucune trace

13/Quels sont les délais d'obtention des résultats? *

immédiatement

24-48h

10-15j

1 mois

ne sait pas 
14/Si vous avez déjà fait une déclaration, comment avez-vous obtenu le plus souvent les résultats? *

$\square$ Par courrier

$\square$ Par mail

$\square$ Par téléphone

$\square$ Je n'ai pas obtenu les résultats

15/Comment avez-vous obtenu le plus souvent les résultats de la consultation d'allergologie *

Par courrier

Par mail

Par téléphone

Je n'ai pas obtenu les résultats

16/Les prélèvements pharmacologiques sont-ils suffisants pour confirmer le diagnostic? *

oui

non

17/La consultation d'allergologique doit être réalisée dans quel délai optimal? *
immédiatement
dans la semaine
4-6semaines
3 mois après
Ne sait pas

18/Par qui le bilan allergologique doit-il être réalisé? *

$\square$ tous les allergologues de ville

$\square$ certains allergologues de ville

$\square$ tous les allergologues hospitaliers

$\square$ certains allergologues hospitaliers

19/Avez vous un allergologue correspondant? *

$\square$ dans votre structure d'exercice

$\square$ en ville

$\square$ au $\mathrm{CHU}$

$\square$ ne sait pas

20/Qui prend rendez-vous avec l'allergologue? *

$\square$ le patient lui même

$\square$ l'infirmière du service de chirurgie

$\square$ le chirurgien

$\square$ vous-même

$\square$ le service de pharmacovigilance

$\square$ l'interne d'anesthésie

$\square$ l'interne de chirurgie

$\square$ Ne sait pas

21/Comment vous assurez-vous que le rendez vous a été bien pris? *

$\square$ en regardant les comptes rendus dans le logiciel de suivi du patient

$\square$ en appelant le secrétariat d'allergologie

$\square$ en appelant le patient

$\square$ en appelant le service de pharmacovigilance

$\square$ vous ne vérifiez pas

22/Si à l'interrogatoire du patient, celui-ci n'est plus capable de préciser son type d'allergie, comment pouvez vous le retrouver? *

$\square$ en regardant les comptes rendus dans le logiciel de suivi patient

$\square$ en regardant les prélèvements sanguins dans le logiciel de biologie du patient

$\square$ en appelant la pharmacovigilance

$\square$ en demandant la carte d'allergie du patient

$\square$ en contactant son médecin traitant

23/ L'adressez vous à un allergologue avant l'intervention? *

Oui

Non

24/Lors de vos consultations de pré-anesthésies chez les patients allergiques, cherchez-vous systématiquement les comptes rendus d'allergologies? ? *

oui

non 


\section{ANNEXE 3: Déclaration en ligne}

\section{COORDONNÉES DU DÉCLARANT}

${ }^{\star}$ Nom :

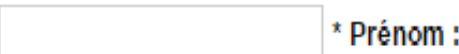

* Téléphone :

Adresse :

Code postal:

Commune:

Email :

Qualifications :

Médecin généraliste

Médecin Spécialiste

Chirurgien-dentiste

Sage-femme

Pharmacien

Autre:

PATIENT TRAITÉ

${ }^{*}$ Nom :

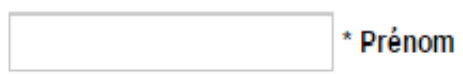

Date de naissance

Age :

Poids :

Taille :

Département

* Sexe: Homme

Femme

S'il s'agit d'un nouveau né, le médicament a été pris

Par le nouveau né

Par la mère durant la grossesse

Par la mère durant l'allaitement

Antécédents / Facteurs favorisants : 
Département de

\section{GRAVITÉ}

Hospitalisation ou prolongation

d'hospitalisation

Autre situation médicale grave
Incapacite ou invalidité

permanente

Non grave
Mise en jeu du pronostic vital

Décès

EVOLUTION

Guérison sans séquelles

Guérison avec séquelles

Sujet non encore rétabli

Décès dû à l'effet

Décès auquel l'effet a pu contribuer

Décès sans rapport avec l'effet

Inconnue

Guérison en cours

DOCUMENTS ANNEXES

Document 1: $\quad$ Choisissez un fichier Aucun fichier choisi

† DESCRIPTION DE L'EFFET INDÉSIRABLE 


\section{ANNEXE 4: Yellow card scheme}

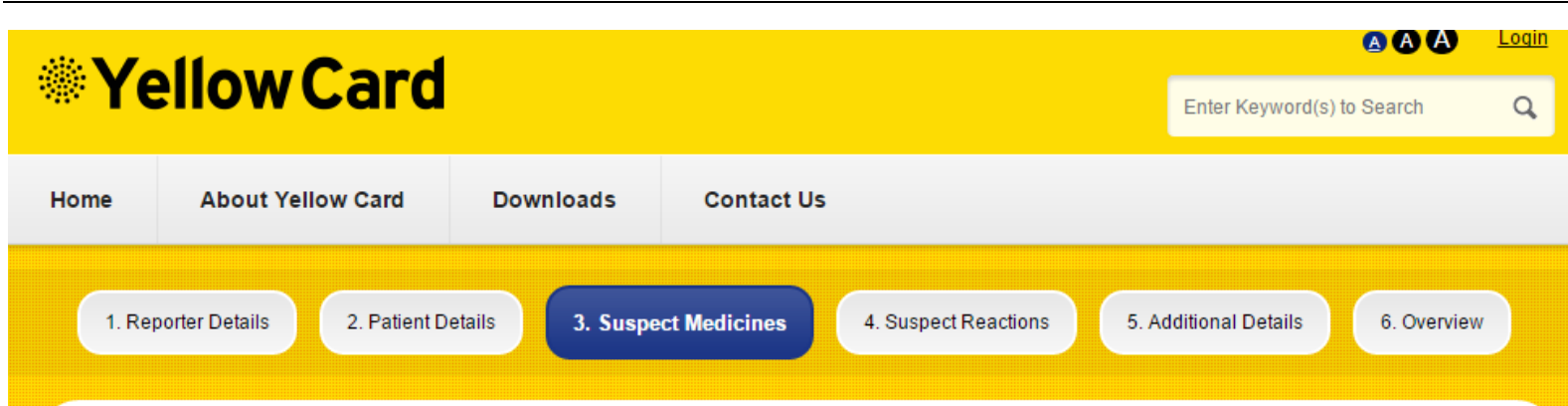

3. Suspect Medicines

i Please provide us with details about the product that has caused your side effect(s). If there is no appropriate brand name available, please enter as much information on the medicine as possible, i.e. Anadin Extra, Zovirax cream, name of herbal product (eg St John's Wort), Simvastatin 10mg tablets etc.

When reporting a suspected ADR to biological medicines and vaccines it is particularly important to provide the brand name and the specific batch number (or confirm that this information is not known).

Fields that you must complete are marked with this symbol: required 
Introduction : Depuis 2001, la SFAR préconise lors d'un choc anaphylactique (CA) une investigation immédiate par un bilan biologique puis une consultation d'allergologie à distance. Depuis 1984, le CA doit être déclaré à la pharmacovigilance. Pourtant, peu de patients bénéficient d'une consultation. L'objectif de l'étude était d'évaluer, à l'échelon régional, la qualité de la déclaration des CA supposés aux curares par les médecins anesthésistesréanimateurs (MAR) et l'accompagnement du patient jusqu'au diagnostic positif d'allergie.

Matériel et méthodes : Il s'agissait d'une étude prospective observationnelle basée sur un questionnaire anonyme envoyé à tous les MAR travaillant en centre hospitalier en Picardie.

Résultats : Le taux de participation était de 64\%. $68 \%$ avaient déjà été confrontés à un CA. $17 \%$ déclaraient toujours les CA tous grades confondus. $11 \%$ déclaraient en ligne. $87 \%$ prélevaient un dosage de tryptase à la $1^{\text {ère }}$ heure et la moitié répétait ce prélèvement à la $2^{\text {ème }}$ heure. $73 \%$ informaient toujours leur patient de l'existence d'une allergie, 53\% évoquaient le produit suspecté. $41 \%$ expliquaient les modalités de la consultation d'allergologie et $23 \%$ lui délivraient une carte provisoire d'allergie. Tous les MAR correspondaient avec un allergologue référent au CHU.

Discussion : La sous-déclaration concernait principalement les CA de grades faibles. Une minorité de MAR déclarait en ligne. Le $1^{\text {er }}$ dosage était correctement effectué mais insuffisamment renouvelé. L'information du patient était insuffisante sur les modalités de la consultation spécialisée. La diffusion de la carte d'allergique doit être optimisée.

Conclusion : Alors que les MAR sont régulièrement confrontés aux CA, il existe encore une sous-déclaration au niveau régional.

Mots clés : anaphylaxie, anesthésie, pharmacovigilance.

Introduction: Since 2001, SFAR recommands an emergent investigation in case of anaphylactic shock (AS), based on blood analysis and subsequent specialized consultation. Since 1984, drug safety statement is mandatory. The aim of this study was to evaluate, at regional level, the quality of reporting of AS linked to neuromuscular blocking agents by anesthesiologists physicians (AP) and the patient orientation to the positive diagnosis of allergy.

Materials and methods: It was a descriptive prospective observational study based on anonymous questionnaires sent to AP working in public hospitals of Picardie.

Results : The participation rate was $64 \% .68 \%$ of AS had already faced with an AS. $17 \%$ always declared AS to drug safety organism whatever its severity. $11 \%$ reported this adverse event with online process. 87\% collected a blood tryptase test within the first hour after AS and half of them repeated it twice. $73 \%$ always informed their patients of the occurrence of an allergy, $53 \%$ explained the suspected drug. $41 \%$ gave details about specialized consultation. $23 \%$ provided the patient a temporary allergy card. All had a referent allergist at the University Hospital.

Discussion : Underreporting concerned mainly low grades of AS. A minority of AP reported the adverse event online. The first blood test was correctly performed but inadequatly renewed. Information given to the patient was insufficiently focused on subsequent allergologic consultation. Distribution of allergic card should be improved.

Conclusion : Whereas AP commonly face with AS, there is still an underreporting of these severe adverse events at regional level.Keywords : anaphylaxis, anesthesia, drug safety 